\title{
LI.
}

\section{Was sind und was sollen die Zahlen?}

[Erste Auflage 1888. Sechste Auflage 1930.]

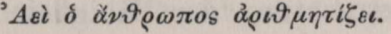

\section{Meiner Schwester}

\section{Julie}

und meinem Bruder

\section{Adolf,}

Dr. jur., Oberlandesgerichtsrat zu Braunschweig in herzlicher Liebe gewidmet.

\section{Vorwort zur ersten Auflage.}

Was beweisbar ist, soll in der Wissenschaft nicht ohne Beweis geglaubt werden. So einleuchtend diese Forderung erscheint, so ist sie doch, wie ich glaube, selbst bei der Begründung der einfachsten Wissenschaft, nämlich desjenigen Teiles der Logik, welcher die Lehre von den Zahlen behandelt, auch nach den neuesten Darstellungen*) noch keineswegs als erfüllt anzusehen. Indem ich die Arithmetik (Algebra, Analysis) nur einen Teil der Logik nenne, spreche ich schon aus, daß ich den Zahlbegriff für gänzlich unabhängig von den Vorstellungen oder Anschauungen des Raumes und der Zeit, daß ich ihn vielmehr für einen unmittelbaren Ausfluß der reinen Denkgesetze halte. Meine Hauptantwort auf die im Titel dieser Schrift gestellte Frage lautet: die Zahlen sind freie Schöpfungen des menschlichen Geistes, sie dienen als ein Mittel, um die Verschiedenheit der Dinge leichter und schärfer aufzufassen. Durch den rein logischen Aufbau der Zahlen-Wissenschaft und durch das in ihr

*) Von den mir bekannt gewordenen Schriften erwähne ich das verdienstvolle Lehrbuch der Arithmetik und Algebra von E. Schröder (Leipzig 1873), in welchem man auch ein Literaturverzeichnis findet, und außerdem die $\mathrm{Ab}$ handlungen von Kronecker und von Helmholtz über den Zahlbegriff und über Zählen und Messen (in der Sammlung der an E. Zeller gerichteten philosophischen Aufsätze, Leipzig 1887). Das Erscheinen dieser Abhandlungen ist die Veranlassung, welche mich bewogen hat, nun auch mit meiner, in mancher Beziehung ähnlichen, aber durch ihre Begründung doch wesentlich verschiedenen Auffassung hervorzutreten, die ich mir seit vielen Jahren und ohne jede Beeinflussung von irgendwelcher Seite gebildet habe. 
gewonnene stetige Zahlen-Reich sind wir erst in den Stand gesetzt, unsere Vorstellungen von Raum und Zeit genau zu untersuchen, indem wir dieselben auf dieses in unserem Geiste geschaffene ZahlenReich beziehen*). Verfolgt man genau, was wir bei dem Zählen der Menge oder Anzahl von Dingen tun, so wird man auf die Betrachtung der Fähigkeit des Geistes geführt, Dinge auf Dinge zu beziehen, einem Dinge ein Ding entsprechen zu lassen, oder ein Ding durch ein Ding abzubilden, ohne welche Fähigkeit überhaupt kein Denken möglich ist. Auf dieser einzigen, auch sonst ganz unentbehrlichen Grundlage muß nach meiner Ansicht, wie ich auch schon bei einer Ankündigung der vorliegenden Schrift ausgesprochen habe**), die gesamte Wissenschaft der Zahlen errichtet werden. Die Absicht einer solchen Darstellung habe ich schon vor der Herausgabe meiner Schrift über die Stetigkeit gefaßt, aber erst nach Erscheinen derselben, und mit vielen Unterbrechungen, die durch gesteigerte Amtsgeschäfte und andere notwendige Arbeiten veranlaßt wurden, habe ich in den Jahren 1872 bis 1878 auf wenigen Blättern einen ersten Entwurf aufgeschrieben, welchen dann mehrere Mathematiker eingesehen und teilweise mit mir besprochen haben. Er trägt denselben Titel und enthält, wenn auch nicht auf das beste geordnet, doch alle wesentlichen Grundgedanken meiner vorliegenden Schrift, die nur deren sorgfältige Ausführung gibt; als solche Hauptpunkte erwähne ich hier die scharfe Unterscheidung des Endlichen vom Unendlichen (64), den Begriff der Anzahl von Dingen (161), den Nachweis, daß die unter dem Namen der vollständigen Induktion (oder des Schlusses von $n$ auf $n+1$ ) bekannte Beweisart wirklich beweiskräftig $(59,60,80)$, und daß auch die Definition durch Induktion (oder Rekursion) bestimmt und widerspruchsfrei ist (126).

Diese Schrift kann jeder verstehen, welcher das besitzt, was man den gesunden Menschenverstand nennt; philosophische oder mathematische Schulkenntnisse sind dazu nicht im geringsten erforderlich. Aber ich weiß sehr wohl, daß gar mancher in den schattenhaften Gestalten, die ich ihm vorführe, seine Zahlen, die ihn als treue und vertraute Freunde durch das ganze Leben begleitet haben, kaum wiedererkennen mag; er wird durch die lange, der

*) Vgl. § 3 meiner Schrift: Stetigkeit und irrationale Zahlen (Braunschweig 1872).

**) Dirichlets Vorlesungen über Zahlentheorie, dritte Auflage, 1879, §163, Anmerkung auf S. 470 . 
Beschaffenheit unseres Treppenverstandes entsprechende Reihe von einfachen Schlüssen, durch die nüchterne Zergliederung der Gedankenreihen, auf denen die Gesetze der Zahlen beruhen, abgeschreckt und ungeduldig darüber werden, Beweise für Wahrheiten verfolgen zu sollen, die ihm nach seiner vermeintlichen inneren Anschauung von vornherein einleuchtend und gewiß erscheinen. Ich erblicke dagegen gerade in der Möglichkeit, solche Wahrheiten auf andere, einfachere zurückzuführen, mag die Reihe der Schlüsse noch so lang und scheinbar künstlich sein, einen überzeugenden Beweis dafür, daß ihr Besitz oder der Glaube an sie niemals unmittelbar durch innere Anschauung gegeben, sondern immer nur durch eine mehr oder weniger vollständige Wiederholung der einzelnen Schlüsse erworben ist. Ich möchte diese, der Schnelligkeit ihrer Ausführung wegen schwer zu verfolgende Denktätigkeit mit derjenigen vergleichen, welche ein vollkommen geübter Leser beim Lesen verrichtet; auch dieses Lesen bleibt immer eine mehr oder weniger vollständige Wiederholung der einzelnen Schritte, welche der Anfänger bei dem mühseligen Buchstabieren auszuführen hat; ein sehr kleiner Teil derselben, und deshalb eine sehr kleine Arbeit oder Anstrengung des Geistes reicht aber für den geübten Leser schon aus, um das richtige, wahre Wort zu erkennen, freilich nur mit sehr großer Wahrscheinlichkeit; denn bekanntlich begegnet es auch dem geübtesten Korrektor von Zeit zu Zeit, einen Druckfehler stehenzulassen, d. h. falsch zu lesen, was unmöglich wäre, wenn die zum Buchstabieren gehörige Gedankenkette vollständig wiederholt würde. So sind wir auch schon von unserer Geburt an beständig und in immer steigendem Maße veranlaßt, Dinge auf Dinge zu beziehen und damit diejenige Fähigkeit des Geistes zu üben, auf welcher auch die Schöpfung der Zahlen beruht; durch diese schon in unsere ersten Lebensjahre fallende unablässige, wenn auch absichtslose Übung und die damit verbundene Bildung von Urteilen und Schlußreihen erwerben wir uns auch einen Schatz von eigentlich arithmetischen Wahrheiten, auf welche später unsere ersten Lehrer sich wie auf etwas Einfaches, Selbstverständliches, in der inneren Anschauung Gegebenes berufen, und so kommt es, daß manche, eigentlich sehr zusammengesetzte Begriffe (wie z. B. der der Anzahl von Dingen) fälschlich für einfach gelten. In diesem Sinne, den ich durch die einem bekannten Spruche nachgebildeten Worte

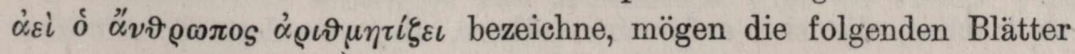


als ein Versuch, die Wissenschaft der Zahlen auf einheitlicher Grundlage zu errichten, wohlwollende Aufnahme finden, und mögen sie andere Mathematiker dazu anregen, die langen Reihen von Schlüssen auf ein bescheideneres, angenehmeres Maß zurückzuführen.

Dem Zwecke dieser Schrift gemäß beschränke ich mich auf die Betrachtung der Reihe der sogenannten natürlichen Zahlen. In welcher Art später die schrittweise Erweiterung des Zahlbegriffes, die Schöpfung der Null, der negativen, gebrochenen, irrationalen und komplexen Zahlen stets durch Zurückführung auf die früheren Begriffe herzustellen ist, und zwar ohne jede Einmischung fremdartiger Vorstellungen (wie z. B. der der meßbaren Größen), die nach meiner Auffassung erst durch die Zahlenwissenschaft zu vollständiger Klarheit erhoben werden können, das habe ich wenigstens an dem Beispiele der irrationalen Zahlen in meiner früheren Schrift über die Stetigkeit (1872) gezeigt; in ganz ähnlicher Weise lassen sich, wie ich daselbst (§3) auch schon ausgesprochen habe, die anderen Erweiterungen leicht behandeln, und ich behalte mir vor, diesem Gegenstande eine zusammenhängende Darstellung zu widmen. Gerade bei dieser Auffassung erscheint es als etwas Selbstverständliches und durchaus nicht Neues, daß jeder auch noch so fern liegende Satz der Algebra und höheren Analysis sich als ein Satz über die natürlichen Zahlen aussprechen läßt, eine Behauptung, die ich auch wiederholt aus dem Munde von Dirichlet gehört habe. Aber ich erblicke keineswegs etwas Verdienstliches darin - und das lag auch Dirichlet gänzlich fern - , diese mühselige Umschreibung wirklich vornehmen und keine anderen als die natürlichen Zahlen benutzen und anerkennen zu wollen. Im Gegenteil, die größten und fruchtbarsten Fortschritte in der Mathematik und anderen Wissenschaften sind vorzugsweise durch die Schöpfung und Einführung neuer Begriffe gemacht, nachdem die häufige Wiederkehr zusammengesetzter Erscheinungen, welche von den alten Begriffen nur mühselig beherrscht werden, dazu gedrängt hat. Über diesen Gegenstand habe ich im Sommer 1854 bei Gelegenheit meiner Habilitation als Privatdozent zu Göttingen einen Vortrag vor der philosophischen Fakultät [LX] zu halten gehabt, dessen Absicht auch von Gauß gebilligt wurde; doch ist hier nicht der Ort, näher darauf einzugehen.

Ich benutze statt dessen die Gelegenheit, noch einige Bemerkungen zu machen, die sich auf meine frühere, oben erwähnte Schrift über 
Stetigkeit und irrationale Zahlen beziehen. Die in ihr vorgetragene, im Herbste 1858 erdachte Theorie der irrationalen Zahlen gründet sich auf diejenige im Gebiete der rationalen Zahlen auftretende Erscheinung ( $\S 4)$, die ich mit dem Namen eines Schnittes belegt und zuerst genau erforscht habe, und sie gipfelt in dem Beweise der Stetigkeit des neuen Gebietes der reellen Zahlen (§5. IV). Sie scheint mir etwas einfacher, ich möchte sagen ruhiger, zu sein als die beiden von ihr und voneinander verschiedenen Theorien, welche von den Herren Weierstraß und G. Cantor aufgestellt sind ond ebenfalls vollkommene Strenge besitzen. Sie ist später ohne wesentliche Änderung von Herrn U. Dini in die Fondamenti per la teorica delle funzioni di variabili reali (Pisa 1878) aufgenommen; aber der Umstand, daß mein Name im Laufe dieser Darstellung nicht bei der Beschreibung der rein arithmetischen Erscheinung des Schnittes, sondern zufällig gerade da erwähnt wird, wo es sich um die Existenz einer dem Schnitte entsprechenden meßbaren Größe handelt, könnte leicht zu der Vermutung führen, daß meine Theorie sich auf die Betrachtung solcher Größen stützte. Nichts könnte unrichtiger sein; vielmehr habe ich in $\S 3$ meiner Schrift verschiedene Gründe angeführt, weshalb ich die Einmischung der meßbaren Größen gänzlich verwerfe, und namentlich am Schlusse hinsichtlich deren Existenz bemerkt, daß für einen großen Teil der Wissenschaft vom Raume die Stetigkeit seiner Gebilde gar nicht einmal eine notwendige Voraussetzung ist, ganz abgesehen davon, daß sie in den Werken über Geometrie zwar wohl dem Namen nach beiläufig erwähnt, aber niemals deutlich erklärt, also auch nicht für Beweise zugänglich gemacht wird. Um dies noch näher zu erläutern, bemerke ich beispielsweise folgendes. Wählt man drei nicht in einer Geraden liegende Punkte $A, B, C$ nach Belieben, nur mit der Beschränkung, daß die Verhältnisse ihrer Entfernungen $A B, A C, B C$ algebraische*) Zahlen sind, und sieht man im Raume nur diejenigen Punkte $\boldsymbol{M}$ als vorhanden an, für welche die Verhältnisse von $A M, B M, C M$ zu $A B$ ebenfalls algebraische Zahlen sind, so ist der aus diesen Punkten $M$ bestehende Raum, wie leicht zu sehen, überall unstetig; aber trotz der Unstetigkeit, Lückenhaftigkeit dieses Raumes sind in ihm, so viel ich sehe, alle Konstruktionen, welche in Euklids Elementen auftreten, genau ebenso

*) Dirichlets Vorlesungen über Zahlentheorie, §159 der zweiten, §160 der dritten Auflage. 
ausführbar wie in dem vollkommen stetigen Raume; die Unstetigkeit dieses Raumes würde daher in Euklids Wissenschaft gar nicht bemerkt, gar nicht empfunden werden. Wenn mir aber jemand sagt, wir könnten uns den Raum gar nicht anders als stetig denken, so möchte ich das bezweifeln und darauf aufmerksam machen, eine wie weit vorgeschrittene, feine wissenschaftliche Bildung erforderlich ist, um nur das Wesen der Stetigkeit deutlich zu erkennen und um zu begreifen, daß außer den rationalen Größenverhältnissen auch irrationale, außer den algebraischen auch transzendente denkbar sind. Um so schöner erscheint es mir, daß der Mensch ohne jede Vorstellung von meßbaren Größen, und zwar durch ein endliches System einfacher Denkschritte sich zur Schöpfung des reinen, stetigen Zahlenreiches aufschwingen kann; und erst mit diesem Hilfsmittel wird es ihm nach meiner Ansicht möglich, die Vorstellung vom stetigen Raume zu einer deutlichen auszubilden.

Dieselbe, auf die Erscheinung des Schnittes gegründete Theorie der irrationalen Zahlen findet man auch dargestellt in der Introduction à la théorie des fonctions d'une variable von J. Tannery (Paris 1886). Wenn ich eine Stelle der Vorrede dieses Werkes richtig verstehe, so hat der Herr Verfasser diese Theorie selbständig, also zu einer Zeit erdacht, wo ihm nicht nur meine Schrift, sondern auch die in derselben Vorrede erwähnten Fondamenti von Dini noch unbekannt waren; diese Übereinstimmung scheint mir ein erfreulicher Beweis dafür zu sein, daß meine Auffassung der Natur der Sache entspricht, was auch von anderen Mathematikern, z. B. von Herrn M. Pasch in seiner Einleitung in die Differential- und Integralrechnung (Leipzig 1883) anerkannt ist. Dagegen kann ich Herrn Tannery nicht ohne weiteres beistimmen, wenn er diese Theorie die Entwicklung eines von Herrn J. Bertrand herrührenden Gedankens nennt, welcher in dessen Traité d'arithmétique enthalten sei und darin bestehe, eine irrationale Zahl zu definieren durch Angabe aller rationalen Zahlen, die kleiner, und aller derjenigen, die größer sind als die zu definierende Zahl. Zu diesem Ausspruch, der von Herrn O. Stolz - wie es scheint, ohne nähere Prüfung - in der Vorrede zum zweiten Teile seiner Vorlesungen über allgemeine Arithmetik (Leipzig 1886) wiederholt ist, erlaube ich mir folgendes zu bemerken. Daß eine irrationale Zahl durch die eben beschriebene Angabe in der Tat als vollständig bestimmt anzusehen ist, diese Überzeugung ist ohne Zweifel auch vor 
Herrn Bertrand immer Gemeingut aller Mathematiker gewesen, die sich mit dem Begriffe des Irrationalen beschäftigt haben; jedem Rechner, der eine irrationale Wurzel einer Gleichung näherungsweise berechnet, schwebt gerade diese Art ihrer Bestimmung vor; und wenn man, wie es Herr Bertrand in seinem Werke ausschließlich tut (mir liegt die achte Auflage aus dem Jahre 1885 vor), die irrationale Zahl als Verhältnis meßbarer Größen auffaßt, so ist diese Art ihrer Bestimmtheit schon auf das deutlichste in der berühmten Definition ausgesprochen, welche Euklid (Elemente V. 5) für die Gleichheit der Verhältnisse aufstellt. Eben diese uralte Überzeugung ist nun gewiß die Quelle meiner Theorie wie derjenigen des Herrn Bertrand und mancher anderen, mehr oder weniger durchgeführten Versuche gewesen, die Einführung der irrationalen Zahlen in die Arithmetik zu begründen. Aber wenn man Herrn Tannery so weit vollständig beistimmen wird, so muß man bei einer wirklichen Prüfung doch sofort bemerken, daß die Darstellung des Herrn Bertrand, in der die Erscheinung des Schnittes in ihrer logischen Reinheit gar nicht einmal erwähnt wird, mit der meinigen durchaus keine Ähnlichkeit hat, insofern sie sogleich ihre Zuflucht zu der Existenz einer meßbaren Größe nimmt, was ich aus den oben besprochenen Gründen gänzlich verwerfe; und abgesehen von diesem Umstande, scheint mir diese Darstellung auch in den nachfolgenden, auf die Annahme dieser Existenz gegründeten Definitionen und Beweisen noch einige so wesentliche Lücken darzubieten, daß ich die in meiner Schrift ( $\S 6)$ ausgesprochene Behauptung, der Satz $\sqrt{2} \cdot \sqrt{3}=\sqrt{6}$ sei noch nirgends streng bewiesen, auch in Hinsicht auf dieses in mancher anderen Beziehung treffliche Werk, welches ich damals noch nicht kannte, für gerechtfertigt halte.

Harzburg, 5. Oktober 1887.

\section{R. Dedekind.}

\section{Vorwort zur zweiten Auflage.}

Die vorliegende Schrift hat bald nach ihrem Erscheinen neben günstigen auch ungünstige Beurteilungen gefunden, ja es sind $\mathrm{ihr}$ arge Fehler vorgeworfen. Ich habe mich von der Richtigkeit dieser Vorwürfe nicht überzeugen können und lasse jetzt die seit kurzem vergriffene Schrift, zu deren öffentlicher Verteidigung es mir an Zeit fehlt, ohne jede Änderung wieder abdrucken, indem ich nur folgende Bemerkungen dem ersten Vorworte hinzufüge. 
Die Eigenschaft, welche ich als Definition (64) des unendlichen Systems benutzt habe, ist schon vor dem Erscheinen meiner Schrift von G. Cantor (Ein Beitrag zur Mannigfaltigkeitslehre, Crelles Journal, Bd. 84; 1878), ja sogar schon von Bolzano (Paradoxien des Unendlichen $\S 20 ; 1851)$ hervorgehoben. Aber keiner der genannten Schriftsteller hat den Versuch gemacht, diese Eigenschaft zur Definition des Unendlichen zu erheben und auf dieser Grundlage die Wissenschaft von den Zahlen streng logisch aufzubauen, und gerade hierin besteht der Inhalt meiner mühsamen Arbeit, die ich in allem Wesentlichen schon mehrere Jahre vor dem Erscheinen der Abhandlung von G. Cantor und zu einer Zeit vollendet hatte, als mir das Werk von Bolzano selbst dem Namen nach gänzlich unbekannt war. Für diejenigen, welche Interesse und Verständnis für die Schwierigkeiten einer solchen Untersuchung haben, bemerke ich noch folgendes. Man kann eine ganz andere Definition des Endlichen und Unendlichen aufstellen, welche insofern noch einfacher erscheint, als bei ihr nicht einmal der Begriff der Ähnlichkeit einer Abbildung (26) vorausgesetzt wird, nämlich:

„Ein System $S$ heißt endlich, wenn es sich so in sich selbst abbilden läßt (36), daß kein echter Teil (6) von $S$ in sich selbst abgebildet wird; im entgegengesetzten Falle heißt $S$ ein unendliches System."

Nun mache man einmal den Versuch, auf dieser neuen Grundlage das Gebäude zu errichten! Man wird alsbald auf große Schwierigkeiten stoßen, und ich glaube behaupten zu dürfen, daß selbst der Nachweis der vollständigen Übereinstimmung ảieser Definition mit der früheren nur dann (und dann auch leicht) gelingt, wenn man die Reihe der natürlichen Zahlen schon als entwickelt ansehen und auch die Schlußbetrachtung in (131) zu Hilfe nehmen darf; und doch ist von allen diesen Dingen weder in der einen noch in der anderen Definition die Rede! Man wird dabei erkennen, wie sehr groß die Anzahl der Gedankenschritte ist, die zu einer solchen Umformung einer Definition erforderlich sind.

Etwa ein Jahr nach der Herausgabe meiner Schrift habe ich die schon im Jahre 1884 erschienenen Grundlagen der Arithmetik von G. Frege kennengelernt. Wie verschieden die in diesem Werke niedergelegte Ansicht über das Wesen der Zahl von der meinigen auch sein mag, so enthält es, namentlich von $\S 79$ an, doch auch sehr nahe Berührungspunkte mit meiner Schrift, insbesondere mit meiner Erklärung (44). Freilich ist die Übereinstimmung wegen der 
abweichenden Ausdrucksweise nicht leicht zu erkennen; aber schon die Bestimmtheit, mit welcher der Verfasser sich über die Schlußweise von $n$ auf $n+1$ ausspricht (unten auf S. 93), zeigt deutlich, daß er hier auf demselben Boden mit mir steht.

Inzwischen sind $(1890-1891)$ die Vorlesungen über die Algebra der Logik von E. Schröder fast vollständig erschienen. Auf die Bedeutung dieses höchst anregenden Werkes, dem ich meine größte Anerkennung zolle, hier näher einzugehen, ist unmöglich; vielmehr möchte ich mich nur entschuldigen, daß ich trotz der auf S. 253 des ersten Teiles gemachten Bemerkung meine etwas schwerfälligen Bezeichnungen (8) und (17) doch beibehalten habe; dieselben machen keinen Anspruch darauf, allgemein angenommen zu werden, sondern bescheiden sich, lediglich den Zwecken dieser arithmetischen Schrift zu dienen, wozu sie nach meiner Ansicht besser geeignet sind, als Summen- und Produktzeichen.

Harzburg, 24. August 1893.

\section{R. Dedekind.}

\section{Vorwort zur dritten Auflage.}

Als ich vor etwa acht Jahren aufgefordert wurde, die damals schon vergriffene zweite Auflage dieser Schrift durch eine dritte zu ersetzen, trug ich Bedenken, darauf einzugehen, weil inzwischen sich Zweifel an der Sicherheit wichtiger Grundlagen meiner Auffassung geltend gemacht hatten. Die Bedeutung und teilweise Berechtigung dieser Zweifel verkenne ich auch heute nicht. Aber mein Vertrauen in die innere Harmonie unserer Logik ist dadurch nicht erschüttert; ich glaube, daß eine strenge Untersuchung der Schöpferkraft des Geistes, aus bestimmten Elementen ein neues Bestimmtes, ihr System zu erschaffen, das notwendig von jedem dieser Elemente verschieden ist, gewiß dazu führen wird, die Grundlagen meiner Schrift einwandfrei zu gestalten. Durch andere Arbeiten bin ich jedoch verhindert, eine so schwierige Untersuchung zu Finde $\mathrm{zu}$ führen, und ich bitte daher um Nachsicht, wenn die Schrift jetzt doch in ungeänderter Form zum dritten Male erscheint, was sich nur dadurch rechtfertigen läßt, daß das Interesse an ihr, wie die anhaltende Nachfrage zeigt, noch nicht erloschen ist.

Braunschweig, 30. September 1911.

R. Dedekind. 
§ 1. Systeme von Elementen.

$\S$ 2. Abbildung eines Systems

4. Abbildung eines Systems in sich selbst Systeme. . . . .

\& 5. Das Endliche und Unendliche

$\S$ 6. Einfach unendliche Systeme. Reihe der natürlichen Zahlen . . . . . 359

$\S$ 7. Größere und kleinere Zahlen. . . . . . . . . . . . . . . . . . 361

$\S$ 8. Endliche und unendliche Teile der Zahlenreihe . . . . . . . . . 368

$\S$ 9. Definition einer Abbildung der Zahlenreihe durch Induktion . . . . . 370

$\S 10$. Die Klasse der einfach unendlichen Systeme. . . . . . . . . . . 376

$\S 11$. Addition der Zahlen ... . . . . . . . . . . . . . . . . . . . 378

$\S 12$. Multiplikation der Zahlen . . . . . . . . . . . . . . . . . 381

$\S 13$. Potenzierung der Zahlen ................... 383

$\S 14$. Anzahl der Elemente eines endlichen Systems . . . . . . . . . . . 384

$\S 1$.

Systeme von Elementen.

1. Im folgenden verstehe ich unter einem Ding jeden Gegenstand unseres Denkens. Um bequem von den Dingen sprechen zu können, bezeichnet man sie durch Zeichen, z. B. durch Buchstaben, und man erlaubt sich, kurz von dem Ding $a$ oder gar von $a$ zu sprechen, wo man in Wahrheit das durch $a$ bezeichnete Ding, keineswegs den Buchstaben $a$ selbst meint. Ein Ding ist vollständig bestimmt durch alles das, was von ihm ausgesagt oder gedacht werden kann. Ein Ding $a$ ist dasselbe wie $b$ (identisch mit $b$ ), und $b$ dasselbe wie $a$, wenn alles, was von $a$ gedacht werden kann, auch von $b$, und wenn alles, was von $b$ gilt, auch von $a$ gedacht werden kann. Daß $a$ und $b$ nur Zeichen oder Namen für ein und dasselbe Ding sind, wird durch das Zeichen $a=b$ und ebenso durch $b=a$ angedeutet. Ist außerdem $b=c$, ist also $c$ ebenfalls, wie $a$, ein Zeichen für das mit $b$ bezeichnete Ding, so ist auch $a=c$. Ist die obige Übereinstimmung des durch $a$ bezeichneten Dinges mit dem durch $b$ bezeichneten Dinge nicht vorhanden, so heißen diese Dinge $a, b$ verschieden, $a$ ist ein anderes Ding wie $b, b$ ein anderes Ding wie $a$; es gibt irgendeine Eigenschaft, die dem einen zukommt, dem anderen nicht zukommt.

2. Es kommt sehr häufig vor, daß verschiedene Dinge $a, b, c \ldots$ aus irgendeiner Veranlassung unter einem gemeinsamen Gesichtspunkte aufgefaßt, im Geiste zusammengestellt werden, und man sagt dann, daß sie ein System $S$ bilden; man nennt die Dinge $a, b, c \ldots$ die Elemente des Systems $S$, sie sind enthalten in $S$; umgekehrt 
besteht $S$ aus diesen Elementen. Ein solches System $S$ (oder ein Inbegriff, eine Mannigfaltigkeit, eine Gesamtheit) ist als Gegenstand unseres Denkens ebenfalls ein Ding (1); es ist vollständig bestimmt, wenn von jedem Ding bestimmt ist, ob es Element von $S$ ist oder nicht*). Das System $S$ ist daher dasselbe wie das System $T$, in Zeichen $S=T$, wenn jedes Element von $S$ auch Element von $T$ und jedes Element von $T$ auch Element von $S$ ist. Für die Gleichförmigkeit der Ausdrucksweise ist es vorteilhaft, auch den besonderen Fall zuzulassen, daß ein System $S$ aus einem einzigen (aus einem und nur einem) Element $a$ besteht, d. h. daß das Ding $a$ Element von $S$, aber jedes von $a$ verschiedene Ding kein Element von $S$ ist. Dagegen wollen wir das leere System, welches gar kein Element enthält, aus gewissen Gründen hier ganz ausschließen, obwohl es für andere Untersuchungen bequem sein kann, ein solches zu erdichten.

3. Erklärung. Ein System $A$ heißt Teil eines Systems $S$, wenn jedes Element von $A$ auch Element von $S$ ist. Da diese Beziehung zwischen einem System $A$ und einem System $S$ im folgenden immer wieder zur Sprache kommen wird, so wollen wir dieselbe zur Abkürzung durch das Zeichen $A 3 S$ ausdrücken. Das umgekehrte Zeichen $S \mathcal{E} A$, wodurch dieselbe Tatsache bezeichnet werden könnte, werde ich der Deutlichkeit und Einfachheit halber gänzlich vermeiden, aber ich werde in Ermangelung eines besseren Wortes bisweilen sagen, daß $S$ Ganzes von $A$ ist, wodurch also ausgedrückt werden soll, daß unter den Elementen von $S$ sich auch alle Elemente von $A$ befinden. Da ferner jedes Element $s$ eines Systems $S$ nach 2 selbst als System aufgefaßt werden kann, so können wir auch hierauf die Bezeichnung $s 3 S$ anwenden.

4. Satz. Zufolge 3 ist $A 3 A$.

5. Satz. Ist $A 3 B$ und $B 3 A$, so ist $A=B$.

Der Beweis folgt aus $3,2$.

*) Auf welche Weise diese Bestimmtheit zustande kommt, und ob wir einen Weg kennen, um hierüber zu entscheiden, ist für alles Folgende gänzlich gleichgültig; die zu entwickelnden allgemeinen Gesetze hängen davon gar nicht ab, sie gelten unter allen Umständen. Ich erwähne dies ausdrücklich, weil Herr Kronecker vor kurzem (im Band 99 des Journals für Mathematik, S. 334 bis 336) der freien Begriffsbildung in der Mathematik gewisse Beschränkungen hat auferlegen wollen, die ich nicht als berechtigt anerkenne; näher hierauf einzugehen, erscheint aber erst dann geboten, wenn der ausgezeichnete Mathematiker seine Gründe für die Notwendigkeit oder auch nur die Zweckmäßigkeit dieser Beschränkungen veröffentlicht haben wird. 
6. Erklärung. Ein System $A$ heißt echter Teil von $S$, wenn $A$ Teil von $S$, aber verschieden von $S$ ist. Nach 5 ist dann $S$ kein Teil von $A$, d. h. (3) es gibt in $S$ ein Element, welches kein Element von $A$ ist.

7. Satz. Ist $A 3 B$ und $B 3 C$, was auch kurz durch $A 3 B 3 C$ bezeichnet werden kann, so ist $A 3 C$, und zwar ist $A$ gewiß echter Teil von $C$, wenn $A$ echter Teil von $B$ oder wenn $B$ echter Teil von $C$ ist.

Der Beweis folgt aus 3,6 .

8. Erklärung. Unter dem aus irgendwelchen Systemen $A, B, C \ldots$ zusammengesetzten System, welches mit $\mathfrak{M}(A, B, C \ldots)$ bezeichnet werden soll, wird dasjenige System verstanden, dessen Elemente durch folgende Vorschrift bestimmt werden: ein Ding gilt dann und nur dann als Element von $\mathfrak{M}(A, B, C \ldots)$, wenn es Element von irgendeinem der Systeme $A, B, C \ldots$, d. h. Element von $A$ oder $B$ oder $C \ldots$ ist. Wir lassen auch den Fall zu, daß nur ein einziges System $A$ vorliegt; dann ist offenbar $\mathfrak{M}(A)=A$. Wir bemerken ferner, daß das aus $A, B, C \ldots$ zusammengesetzte System $\mathfrak{M}(A, B, C \ldots)$ wohl zu unterscheiden ist von demjenigen System, dessen Elemente die Systeme $A, B, C \ldots$ selbst sind.

9. Satz. Die Systeme $A, B, C \ldots$ sind Teile von $\mathfrak{M}(A, B, C \ldots)$.

Der Beweis folgt aus 8,3 .

10. Satz. Sind $A, B, C \ldots$ Teile eines Systems $S$, so ist $\mathfrak{M}(A, B, C \ldots) 3 S$.

Der Beweis folgt aus 8, 3 .

11. Satz. Ist $P$ Teil von einem der Systeme $A, B, C \ldots$, so ist $P 3 \mathfrak{M}(A, B, C \ldots)$.

Der Beweis folgt aus 9,7 .

12. Satz. Ist jedes der Systeme $P, Q \ldots$ Teil von einem der Systeme $A, B, C \ldots$, so ist $\mathfrak{M}(P, Q \ldots)\} \mathfrak{M}(A, B, C \ldots)$.

Der Beweis folgt aus 11, 10.

13. Satz. Ist $A$ zusammengesetzt aus irgendwelchen der Systeme $P, Q \ldots$, so ist $A 3 \mathfrak{M}(P, Q \ldots)$.

Beweis. Denn jedes Element von $A$ ist nach 8 Element von einem der Systeme $P, Q \ldots$, folglich nach 8 auch Element von $\mathfrak{M}(P, Q \ldots)$, woraus nach 3 der Satz folgt.

14. Satz. Ist jedes der Systeme $A, B, C \ldots$ zusammengesetzt aus irgendwelchen der Systeme $P, Q \ldots$, so ist

$$
\mathfrak{M}(A, B, C \ldots) 3 \mathfrak{M}(P, Q \ldots) \text {. }
$$

Der Beweis folgt aus 13, 10. 
15. Satz. Ist jedes der Systeme $P, Q \ldots$ Teil von einem der Systeme $A, B, C \ldots$, und ist jedes der letzteren zusammengesetzt aus irgendwelchen der ersteren, so ist

$$
\mathfrak{M}(P, Q \ldots)=\mathfrak{M}(A, B, C \ldots) \text {. }
$$

Der Beweis folgt aus $12,14,5$.

16. Satz. Ist $A=\mathfrak{M}(P, Q)$ und $B=\mathfrak{M}(Q, R)$, so ist $\mathfrak{M}(A, R)=\mathfrak{M}(P, B)$.

Beweis. Denn nach dem vorhergehenden Satze 15 ist sowohl $\mathfrak{M}(A, R)$ als $\mathfrak{M}(P, B)=\mathfrak{M}(P, Q, R)$.

17. Erklärung. Ein Ding $g$ heißt gemeinsames Element der Systeme $A, B, C \ldots$, wenn es in jedem dieser Systeme (also in $A$ und in $B$ und in $C \ldots$ ) enthalten ist. Ebenso heißt ein System $T$ ein Gemeinteil von $A, B, C \ldots$, wenn $T$ Teil von jedem dieser Systeme ist, und unter der Gemeinheit der Systeme $A, B, C \ldots$ verstehen wir das vollständig bestimmte System $\mathfrak{G}(A, B, C \ldots)$, welches aus allen gemeinsamen Elementen $g$ von $A, B, C \ldots$ besteht und folglich ebenfalls ein Gemeinteil derselben Systeme ist. Wir lassen auch wieder den Fall zu, daß nur ein einziges System $A$ vorliegt; dann ist $\mathfrak{G}(A)=A$ zu setzen. Es kann aber auch der Fall eintreten, daß die Systeme $A, B, C \ldots$ gar kein gemeinsames Element, also auch keinen Gemeinteil, keine Gemeinheit besitzen; sie heißen dann Systeme ohne Gemeinteil, und das Zeichen $(5(A, B, C \ldots)$ ist bedeutungslos (vgl. den Schluß von 2). Wir werden es aber fast immer dem Leser überlassen, bei Sätzen über Gemeinheiten die Bedingung ihrer Existenz hinzuzudenken und die richtige Deutung dieser Sätze auch für den Fall der Nicht-Existenz zu finden.

18. Satz. Jeder Gemeinteil von $A, B, C \ldots$ ist Teil von $(5(A, B, C \ldots)$.

Der Beweis folgt aus 17.

19. Satz. Jeder Teil von $\mathfrak{G}(A, B, C \ldots)$ ist Gemeinteil von $A, B, C \ldots$

Der Beweis folgt aus 17, 7 .

20. Satz. Ist jedes der Systeme $A, B, C \ldots$ Ganzes (3) von einem der Systeme $P, Q \ldots$, so ist

$$
\mathfrak{G}(P, Q \ldots)) 3 \mathfrak{5}(A, B, C \ldots) \text {. }
$$

Beweis. Denn jedes Element von $(\mathscr{C}(P, Q \ldots)$ ist gemeinsames Element von $P, Q \ldots$, also auch gemeinsames Element von $A, B, C \ldots$, w. z. b.w. 
$\S 2$.

Abbildung eines Systems.

21. Erklärung*). Unter einer Abbildung $\varphi$ eines Systems $\boldsymbol{S}$ wird ein Gesetz verstanden, nach welchem zu jedem bestimmten Element $s$ von $S$ ein bestimmtes Ding gehört, welches das Bild von $s$ heißt und mit $\varphi(s)$ bezeichnet wird; wir sagen auch, daß $\varphi(s)$ dem Element $s$ entspricht, daß $\varphi(s)$ durch die Abbildung $\varphi$ aus $s$ entsteht oder erzeugt wird, daß $s$ durch die Abbildung $\varphi$ in $\varphi(s)$ übergeht. Ist nun $T$ irgendein Teil von $S$, so ist in der Abbildung $\varphi$ von $S$ zugleich eine bestimmte Abbildung von $T$ enthalten, welche der Einfachheit wegen wohl mit demselben Zeichen $\varphi$ bezeichnet werden darf and darin besteht, daß jedem Elemente $\boldsymbol{t}$ des Systems $T$ dasselbe Bild $\varphi(t)$ entspricht, welches $t$ als Element von $S$ besitzt; zugleich soll das System, welches aus allen Bildern $\varphi(t)$ besteht, das Bild von $T$ heißen und mit $\varphi(T)$ bezeichnet werden, wodurch auch die Bedeutung von $\varphi(S)$ erklärt ist. Als ein Beispiel einer Abbildung eines Systems ist schon die Belegung seiner Elemente mit bestimmten Zeichen oder Namen anzusehen. Die einfachste Abbildung eines Systems ist diejenige, durch welche jedes seiner Elemente in sich selbst übergeht; sie soll die identische Abbildung des Systems heißen. Der Bequemlichkeit halber wollen wir in den folgenden Sätzen 22, 23, 24, die sich auf eine beliebige Abbildung $\varphi$ eines beliebigen Systems $S$ beziehen, die Bilder von Elementen $s$ und Teilen $T$ entsprechend durch $s^{\prime}$ und $T^{\prime}$ bezeichnen; außerdem setzen wir fest, daß kleine und große lateinische Buchstaben ohne Akzent immer Elemente und Teile dieses Systems $S$ bedeuten sollen.

22. Satz**). Ist $A 3 B$, so ist $A^{\prime} 3 B^{\prime}$.

Beweis. Denn jedes Element von $A^{\prime}$ ist das Bild eines in $A$, also auch in $B$ enthaltenen Elementes und ist folglich Element von $B^{\prime}$, w. z. b. w.

23. Satz. Das Bild von $\mathfrak{M}(A, B, C \ldots)$ ist $\mathfrak{M}\left(A^{\prime}, B^{\prime}, C^{\prime} \ldots\right)$.

Beweis. Bezeichnet man das System $\mathfrak{M}(A, B, C \ldots)$, welches nach 10 ebenfalls Teil von $S$ ist, mit $\boldsymbol{M}$, so ist jedes Element seines Bildes $M^{\prime}$ das Bild $m^{\prime}$ eines Elementes $m$ von $M$; da nun $m$

*) Vgl. Dirichlets Vorlesungen über Zahlentheorie, dritte Auflage, 1879, § 163.

**) Vgl. Satz 27. 
nach 8 auch Element von einem der Systeme $A, B, C \ldots$, und folglich $m^{\prime}$ Element von einem der Systeme $A^{\prime}, B^{\prime}, C^{\prime} \ldots$, also nach 8 auch Element von $\mathfrak{M}\left(A^{\prime}, B^{\prime}, C^{\prime} \ldots\right)$ ist, so ist nach 3

$$
M^{\prime} 3 \mathfrak{M t}\left(A^{\prime}, B^{\prime}, C^{\prime} \ldots\right) \text {. }
$$

Andererseits, da $A, B, C \ldots$ nach 9 Teile von $M$, also $A^{\prime}, B^{\prime}, C^{\prime} \ldots$ nach 22 Teile von $M^{\prime}$ sind, so ist nach 10 auch

$$
\mathfrak{M}\left(A^{\prime}, B^{\prime}, C^{\prime} \ldots\right) 3 M^{\prime}
$$

und hieraus in Verbindung mit dem Obigen folgt nach 5 der zu beweisende Satz

$$
M^{\prime}=\mathfrak{M}\left(A^{\prime}, B^{\prime}, C^{\prime} \ldots\right)
$$

24. Satz*). Das Bild jedes Gemeinteils von $A, B, C \ldots$, also auch das der Gemeinheit $\mathfrak{G}(A, B, C \ldots)$, ist Teil von $\mathfrak{G}\left(A^{\prime}, B^{\prime}, C^{\prime} \ldots\right)$.

Beweis. Denn dasselbe ist nach 22 Gemeinteil von $A^{\prime}, B^{\prime}, C^{\prime} \ldots$, woraus der Satz nach 18 folgt.

25. Erklärung und Satz. Ist $\varphi$ eine Abbildung eines Systems $S$, und $\psi$ eine Abbildung des Bildes $S^{\prime}=\varphi(S)$, so entspringt hieraus immer eine aus $\varphi$ und $\psi$ zusammengesetzte $\left.{ }^{* *}\right)$ Abbildung $\theta$ von $S$, welche darin besteht, daß jedem Elemente $s$ von $S$ das Bild

$$
\theta(s)=\psi\left(s^{\prime}\right)=\psi(\varphi(s))
$$

entspricht, wo wieder $\varphi(s)=s^{\prime}$ gesetzt ist. Diese Abbildung $\theta$ kann kurz durch das Symbol $\psi \cdot \varphi$ oder $\psi \varphi$, das Bild $\theta(s)$ durch $\psi \varphi(s)$ bezeichnet werden, wobei auf die Stellung der Zeichen $\varphi, \psi$ wohl zu achten ist, weil das Zeichen $\varphi \psi$ im allgemeinen bedeutungslos ist und nur dann einen Sinn hat, wenn $\psi\left(S^{\prime}\right) 3 S$ ist. Bedeutet nun $\chi$ eine Abbildung des Systems $\psi\left(S^{\prime}\right)=\psi \varphi(S)$, und $\eta$ die aus $\psi$ und $\chi$ zusammengesetzte Abbildung $\chi \psi$ des Systems $S^{\prime}$, so ist $\chi \theta(s)=\chi \psi\left(s^{\prime}\right)=\eta\left(s^{\prime}\right)=\eta \varphi(s)$, also stimmen die zusammengesetzten Abbildungen $\chi \theta$ und $\eta \varphi$ für jedes Element $s$ von $S$ miteinander überein, d.h. es ist $\chi \theta=\eta \varphi$. Dieser Satz kann nach der Bedeutung von $\theta$ und $\eta$ füglich durch

$$
\chi \cdot \psi \varphi=\chi \psi \cdot \varphi
$$

ausgedrückt, und diese aus $\varphi, \psi, \chi$ zusammengesetzte Abbildung kann kurz durch $\chi \psi \varphi$ bezeichnet werden.

*) Vgl. Satz 29.

**) Eine Verwechslung dieser Zusammensetzung von Abbildungen mit derjenigen der Systeme von Elementen (8) ist wohl nicht zu befürchten. 
$\S 3$.

Ähnlichkeit einer Abbildung. Ähnliche Systeme.

26. Erklärung. Eine Abbildung $\varphi$ eines Systems $S$ heißt ähnlich (oder deutlich), wenn verschiedenen Elementen $a, b$ des Systems $S$ stets verschiedene Bilder $a^{\prime}=\varphi(a), b^{\prime}=\varphi(b)$ entsprechen. Da in diesem Falle umgekehrt aus $s^{\prime}=t^{\prime}$ stets $s=t$ folgt, so ist jedes Element des Systems $S^{\prime}=\varphi(S)$ das Bild $s^{\prime}$ von einem einzigen, vollständig bestimmten Elemente $s$ des Systems $S$, und man kann daher der Abbildung $\varphi$ von $S$ eine umgekehrte, etwa mit $\bar{\varphi}$ zu bezeichnende Abbildung des Systems $S^{\prime}$ gegenüberstellen, welche darin besteht, daß jedem Elemente $s^{\prime}$ von $S^{\prime}$ das Bild $\bar{\varphi}\left(s^{\prime}\right)=s$ entspricht, und offenbar ebenfalls ähnlich ist. Es leuchtet ein, daß $\bar{\varphi}\left(S^{\prime}\right)=S$, daß ferner $\varphi$ die zu $\bar{\varphi}$ gehörige umgekehrte Abbildung, und daß die nach 25 aus $\varphi$ und $\bar{\varphi}$ zusammengesetzte Abbildung $\bar{\varphi} \varphi$ die identische Abbildung von $S$ ist (21). Zugleich ergeben sich folgende Ergänzungen zu $\S 2$ unter Beibehaltung der dortigen Bezeichnungen.

27. Satz*). Ist $A^{\prime} 3 B^{\prime}$, so ist $A 3 B$.

Beweis. Denn wenn $a$ ein Element von $A$, so ist $a^{\prime}$ ein Element von $A^{\prime}$, also auch von $B^{\prime}$, mithin $=b^{\prime}$, wo $b$ ein Element von $B$; da aber aus $a^{\prime}=b^{\prime}$ immer $a=b$ folgt, so ist jedes Element $a$ von $A$ auch Element von $B$, w. z. b. w.

28. Satz. Ist $A^{\prime}=B^{\prime}$, so ist $A=B$.

Der Beweis folgt aus 27, 4, 5 .

29. Satz**). Ist $G=\mathfrak{G}(A, B, C \ldots)$, so ist $G^{\prime}=\mathfrak{G}\left(A^{\prime}, B^{\prime}, C^{\prime} \ldots\right)$.

Beweis. Jedes Element von $\left(5\left(A^{\prime}, B^{\prime}, C^{\prime} \ldots\right)\right.$ ist jedenfalls in $S^{\prime}$ enthalten, also das Bild $g^{\prime}$ eines in $S$ enthaltenen Elementes $g$; da aber $g^{\prime}$ gemeinsames Element von $A^{\prime}, B^{\prime}, C^{\prime} \ldots$ ist, so muß $g$ nach 27 gemeinsames Element von $A, B, C \ldots$, also auch Element von $G$ sein; mithin ist jedes Element von $\left(\mathfrak{G}\left(A^{\prime}, B^{\prime}, C^{\prime} \ldots\right)\right.$ Bild eines Elementes $g$ von $G$, also Element von $G^{\prime}$, d. h. es ist $\left(\mathfrak{5}\left(A^{\prime}, B^{\prime}, C^{\prime} \ldots\right) 3 G^{\prime}\right.$, und hieraus folgt unser Satz mit Rücksicht auf 24, 5.

30. Satz. Die identische Abbildung eines Systems ist immer eine ähnliche Abbildung.

31. Satz. Ist $\varphi$ eine ähnliche Abbildung von $S$, und $\psi$ eine ähnliche Abbildung von $\varphi(S)$, so ist die aus $\varphi$ und $\psi$ zusammen-

*) Vgl. Satz 22.

**) Vgl. Satz 24. 
gesetzte Abbildung $\psi \varphi$ von $S$ ebenfalls eine ähnliche, und die zugehörige umgekehrte Abbildung $\overline{\psi \varphi}$ ist $=\bar{\varphi} \bar{\psi}$.

Beweis. Denn verschiedenen Elementen $a, b$ von $S$ entsprechen verschiedene Bilder $a^{\prime}=\varphi(a), b^{\prime}=\varphi(b)$, und diesen wieder verschiedene Bilder $\psi\left(a^{\prime}\right)=\psi \varphi(a), \psi\left(b^{\prime}\right)=\psi \varphi(b)$, also ist $\psi \varphi$ eine ähnliche Abbildung. Außerdem geht jedes Element $\psi \varphi(s)=\psi\left(s^{\prime}\right)$ des Systems $\psi \varphi(S)$ durch $\bar{\psi}$ in $s^{\prime}=\varphi(s)$ und dieses durch $\bar{\varphi}$ in $s$ über, also geht $\psi \varphi(s)$ durch $\bar{\varphi} \bar{\psi}$ in $s$ über, w. z. b. w.

32. Erklärung. Die Systeme $R, S$ heißen ähnlich, wenn es eine derartige ähnliche Abbildung $\varphi$ von $S$ gibt, daß $\varphi(S)=R$, also auch $\bar{\varphi}(R)=S$ wird. Offenbar ist nach 30 jedes System sich selbst ähnlich.

33. Satz. Sind $R, S$ ähnliche Systeme, so ist jedes mit $R$ ähnliche System $Q$ auch mit $S$ ähnlich.

Beweis. Denn sind $\varphi, \psi$ solche ähnliche Abbildungen von $S, R$, daß $\varphi(S)=R, \psi(R)=Q$ wird, so ist (nach 31) $\psi \varphi$ eine solche ähnliche Abbildung von $S$, daß $\psi \varphi(S)=Q$ wird, w. z. b. w.

34. Erklärung. Man kann daher alle Systeme in Klassen einteilen, indem man in eine bestimmte Klasse alle und nur die Systeme $Q, R, S \ldots$ aufnimmt, welche einem bestimmten System $R$, dem Repräsentanten der Klasse, ähnlich sind; nach dem vorhergehenden Satze 33 ändert sich die Klasse nicht, wenn irgendein anderes ihr angehöriges System $S$ als Repräsentant gewählt wird.

35. Satz. Sind $R, S$ ähnliche Systeme, so ist jeder Teil von $S$ auch einem Teile von $R$, jeder echte Teil von $S$ auch einem echten Teile von $R$ ähnlich.

Beweis. Denn wenn $\varphi$ eine ähnliche Abbildung von $S$, $\varphi(S)=R$, und $T 3 S$ ist, so ist nach 22 das mit $T$ ähnliche System $\varphi(T) 3 R$; ist ferner $T$ echter Teil von $S$, und $s$ ein nicht in $T$ enthaltenes Element von $S$, so kann das in $R$ enthaltene Element $\varphi(s)$ nach 27 nicht in $\varphi(T)$ enthalten sein; mithin ist $\varphi(T)$ ochter Teil von $R$, w. z. b.w.

\section{$\S 4$.}

Abbildung eines Systems in sich selbst.

36. Erklärung. Ist $\varphi$ eine ähnliche oder unähnliche Abbildung eines Systems $S$, und $\varphi(S)$ Teil eines Systems $Z$, so nennen wir $\varphi$ eine Abbildung von $S$ in $Z$, und wir sagen, $S$ werde durch $\varphi$ in $Z$ 
abgebildet. Wir nennen daher $\varphi$ eine Abbildung des Systems $S$ in sich selbst, wenn $\varphi(S) 3 S$ ist, und wir wollen in diesem Paragraphen die allgemeinen Gesetze einer solchen Abbildung $\varphi$ untersuchen. Hierbei bedienen wir uns derselben Bezeichnungen wie in $\S 2$, indem wir wieder $\varphi(s)=s^{\prime}, \varphi(T)=T^{\prime}$ setzen. Diese Bilder $s^{\prime}, T^{\prime}$ sind zufolge 22,7 jetzt selbst wieder Elemente oder Teile von $S$, wie alle mit lateinischen Buchstaben bezeichneten Dinge.

37. Erklärung. $K$ heißt eine Kette, wenn $K^{\prime} 3 K$ ist. Wir bemerken ausdrücklich, daß dieser Name dem Teile $K$ des Systems $S$ nicht etwa an sich zukommt, sondern nur in Beziehung auf die bestimmte Abbildung $\varphi$ erteilt wird; in bezug auf eine andere Abbildung des Systems $S$ in sich selbst kann $K$ sehr wohl keine Kette sein.

38. Satz. $S$ ist eine Kette.

39. Satz. Das Bild $K^{\prime}$ einer Kette $K$ ist eine Kette.

Bew eis. Denn aus $K^{\prime} 3 K$ folgt nach 22 auch $\left(K^{\prime}\right)^{\prime} 3 K^{\prime}$, w. z. b. w.

40. Satz. Ist $A$ Teil einer Kette $K$, so ist auch $A^{\prime} 3 K$.

Beweis. Denn aus $A 3 K$ folgt (nach 22) $A^{\prime} 3 K^{\prime}$, und da (nach 37 ) $K^{\prime} 3 K$ ist, so folgt (nach 7) $A^{\prime} 3 K$, w. z. b. w.

41. Satz. Ist das Bild $A^{\prime}$ Teil einer Kette $L$, so gibt es eine Kette $K$, welche den Bedingungen $A 3 K, K^{\prime} 3 L$ genügt; und zwar ist $\mathfrak{M}(A, L)$ eine solche Kette $K$.

Beweis. Setzt man wirklich $K=\mathfrak{M}(A, L)$, so ist nach 9 die eine Bedingung $A 3 K$ erfüllt. Da nach 23 ferner $K^{\prime}=\mathfrak{M}\left(A^{\prime}, L^{\prime}\right)$ und nach Annahme $A^{\prime} 3 L, L^{\prime} 3 L$ ist, so ist nach 10 auch die andere Bedingung $K^{\prime} 3 L$ erfüllt, und hieraus folgt, weil (nach 9) $L 3 K$ ist, auch $K^{\prime} 3 K$, d. h. $K$ ist eine Kette, w. z. b. w.

42. Satz. Ein aus lauter Ketten $A, B, C \ldots$ zusammengesetztes System $M$ ist eine Kette.

Beweis. Da (nach 23) $M^{\prime}=\mathfrak{M}\left(A^{\prime}, B^{\prime}, C^{\prime} \ldots\right)$ und nach Annahme $A^{\prime} 3 A, B^{\prime} 3 B, C^{\prime} 3 C \ldots$ ist, so folgt (nach 12) $M^{\prime} 3 M$, w. z. b. w.

43. Satz. Die Gemeinheit $G$ von lauter Ketten $A, B, C \ldots$ ist eine Kette.

Beweis. Da $G$ nach 17 Gemeinteil von $A, B, C \ldots$, also $G^{\prime}$ nach 22 Gemeinteil von $A^{\prime}, B^{\prime}, C^{\prime} \ldots$ und nach Annahme $A^{\prime} 3 A$, $B^{\prime} 3 B, C^{\prime} 3 C \ldots$ ist, so ist (nach 7) $G^{\prime}$ auch Gemeinteil von $A, B, C \ldots$ und folglich nach 18 auch Teil von $G$, w. z. b. w. 
44. Erklärung. Ist $A$ irgendein Teil von $S$, so wollen wir mit $A_{0}$ die Gemeinheit aller derjenigen Ketten (z. B. $S$ ) bezeichnen, von welchen $A$ Teil ist; diese Gemeinheit $A_{0}$ existiert (vgl. 17), weil ja $A$ selbst Gemeinteil aller dieser Ketten ist. Da ferner $A_{0}$ nach 43 eine Kette ist, so wollen wir $A_{0}$ die Kette des Systems $A$ oder kurz die Kette von $A$ nennen. Auch diese Erklärung bezieht sich durchaus auf die zugrunde liegende bestimmte Abbildung $\varphi$ des Systems $S$ in sich selbst, und wenn es später der Deutlichkeit wegen nötig wird, so wollen wir statt $A_{0}$ lieber das Zeichen $\varphi_{0}(A)$ setzen, und ebenso werden wir die einer anderen Abbildung $\omega$ entsprechende Kette von $A$ mit $\omega_{0}(A)$ bezeichnen. Es gelten nun für diesen sehr wichtigen Begriff die folgenden Sätze.

45. Satz. Es ist $A 3 A_{0}$.

Beweis. Denn $A$ ist Gemeinteil aller derjenigen Ketten, deren Gemeinheit $A_{0}$ ist, woraus der Satz nach 18 folgt.

46. Satz. Es ist $\left(A_{0}\right)^{\prime} 3 A_{0}$.

Beweis. Denn nach 44 ist $A_{0}$ eine Kette (37).

47. Satz. Ist $A$ Teil einer Kette $K$, so ist auch $A_{0} 3 K$.

Beweis. Denn $A_{0}$ ist die Gemeinheit und folglich auch ein Gemeinteil aller der Ketten $K$, von denen $A$ Teil ist.

48. Bemerkung. Man überzeugt sich leicht, daß der in 44 erklärte Begriff der Kette $A_{0}$ durch die vorstehenden Sätze 45, 46, 47 vollständig charakterisiert ist.

49. Satz. Es ist $A^{\prime} 3\left(A_{0}\right)^{\prime}$.

Der Beweis folgt aus $45,22$.

50. Satz. Es ist $A^{\prime} 3 A_{0}$.

Der Beweis folgt aus $49,46,7$.

51. Satz. Ist $A$ eine Kette, so ist $A_{0}=A$.

Beweis. Da $A$ Teil der Kette $A$ ist, so ist nach 47 auch $A_{0} 3 A$, woraus nach 45,5 der Satz folgt.

52. Satz. Ist $B 3 A$, so ist $B 3 A_{0}$.

Der Beweis folgt aus 45,7 .

53. Satz. Ist $B 3 A_{0}$, so ist $B_{0} 3 A_{0}$, und umgekehrt.

Beweis. Weil $A_{0}$ eine Kette ist, so folgt nach 47 aus $B 3 A_{0}$ auch $B_{0} 3 A_{0}$; umgekehrt, wenn $B_{0} 3 A_{0}$, so folgt nach 7 auch $B 3 A_{0}$, weil (nach 45$) B 3 B_{0}$ ist.

54. Satz. Ist $B 3 A$, so ist $B_{0} 3 A_{0}$.

Der Beweis folgt aus 52, 53. 
55. Satz. Ist $B 3 A_{0}$, so ist auch $B^{\prime} 3 A_{0}$.

Beweis. Denn nach 53 ist $B_{0} 3 A_{0}$, und da (nach 50) $B^{\prime} 3 B_{0}$ ist, so folgt der zu beweisende Satz aus 7 . Dasselbe ergibt sich, wie leicht zu sehen, auch aus $22,46,7$ oder auch aus 40 .

56. Satz. Ist $B 3 A_{0}$, so ist $\left(B_{0}\right)^{\prime} 3\left(A_{0}\right)^{\prime}$.

Der Beweis folgt aus 53, 22.

57. Satz und Erklärung. Es ist $\left(A_{0}\right)^{\prime}=\left(A^{\prime}\right)_{0}$, d. h. das Bild der Kette von $A$ ist zugleich die Kette des Bildes von $A$. Man kann daher dieses System kurz durch $A_{0}^{\prime}$ bezeichnen und nach Belieben das Kettenbild oder die Bildkette von $A$ nennen. Nach der deutlicheren in 44 angegebenen Bezeichnung würde der Satz durch $\varphi\left(\varphi_{0}(A)\right)=\varphi_{0}(\varphi(A))$ auszudrücken sein.

Beweis. Setzt man zur Abkürzung $\left(A^{\prime}\right)_{0}=L$, so ist $L$ eine Kette (44), und nach 45 ist $A^{\prime} 3 L$, mithin gibt es nach 41 eine Kette $K$, welche den Bedingungen $A 3 K, K^{\prime} 3 L$ genügt; hieraus folgt nach 47 auch $A_{0} 3 K$, also $\left(A_{0}\right)^{\prime} 3 K^{\prime}$, und folglich nach 7 auch $\left(A_{0}\right)^{\prime} 3 L$, d. h.

$$
\left(A_{0}\right)^{\prime} 3\left(A^{\prime}\right)_{0} \text {. }
$$

Da nach 49 ferner $A^{\prime} 3\left(A_{0}\right)^{\prime}$ und $\left(A_{0}\right)^{\prime}$ nach 44,39 eine Kette ist, so ist nach 47 auch

$$
\left(A^{\prime}\right)_{0} 3\left(A_{0}\right)^{\prime}
$$

woraus in Verbindung mit dem obigen Ergebnis der zu beweisende Satz folgt (5).

58. Satz. Es ist $A_{0}=\mathfrak{M}\left(A, A_{0}^{\prime}\right)$, d. h. die Kette von $A$ ist zusammengesetzt aus $A$ und der Bildkette von $A$.

Beweis. Setzt man zur Abkürzung wieder

$$
L=A_{0}^{\prime}=\left(A_{0}\right)^{\prime}=\left(A^{\prime}\right)_{0} \text { und } K=\mathfrak{M}(A, L),
$$

so ist (nach 45) $A^{\prime} 3 L$, und da $L$ eine Kette ist, so gilt nach 41 dasselbe von $K$; da ferner $A 3 K$ ist (9), so folgt nach 47 auch

$$
A_{0} 3 K \text {. }
$$

Andererseits, da (nach 45) $A 3 A_{0}$ und nach 46 auch $L 3 A_{0}$, so ist nach 10 auch

$$
\text { K } 3 A_{0} \text {, }
$$

woraus in Verbindung mit dem obigen Ergebnis der zu beweisende Satz $A_{0}=K$ folgt (5).

59. Satz der vollständigen Induktion. Um zu beweisen, daß die Kette $A_{0}$ Teil irgendeines Systems $\Sigma$ ist - mag letzteres Teil von $S$ sein oder nicht -, genügt es zu zeigen,

Q. daß $A 3 \Sigma$, und 
б. daß das Bild jedes gemeinsamen Elementes von $A_{0}$ und $\Sigma$ ebenfalls Element von $\Sigma$ ist.

Beweis. Denn wenn $\varrho$ wahr ist, so existiert nach 45 jedenfalls die Gemeinheit $G=\mathfrak{G}\left(A_{0}, \Sigma\right)$, und zwar ist (nach 18) $A 3 G$; da außerdem nach 17

$$
\text { G } 3 A_{0}
$$

ist, so ist $G$ auch Teil unseres Systems $S$, welches durch $\varphi$ in sich selbst abgebildet ist, und zugleich folgt nach 55 auch $G^{\prime} 3 A_{0}$. Wenn nun $\sigma$ ebenfalls wahr, d. h. wenn $G^{\prime} 3 \Sigma$ ist, so muß $G^{\prime}$ als Gemeinteil der Systeme $A_{0}, \Sigma$ nach 18 Teil ihrer Gemeinheit $G$ sein, d. h. $G$ ist eine Kette (37), und da, wie schon oben bemerkt, $A 3 G$ ist, so folgt nach 47 auch

$$
A_{0} 3 G
$$

und hieraus in Verbindung mit dem obigen Ergebnis $G=A_{0}$, also nach 17 auch $A_{0} 3 \Sigma$, w. z. b. w.

60. Der vorstehende Satz bildet, wie sich später zeigen wird, die wissenschaftliche Grundlage für die unter dem Namen der vollständigen Induktion (des Schlusses von $n$ auf $n+1$ ) bekannte Beweisart, und er kann auch auf folgende Weise ausgesprochen werden: Um zu beweisen, daß alle Elemente der Kette $A_{0}$ eine gewisse Eigenschaft $\mathbb{E}$ besitzen (oder daß ein Satz $\mathfrak{S}$, in welchem von einem unbestimmten Dinge $n$ die Rede ist, wirklich für alle Elemente $n$ der Kette $A_{0}$ gilt), genügt es zu zeigen,

@. daß alle Elemente $a$ des Systems $A$ die Eigenschaft $\leftleftarrows$ besitzen (oder daß 5 für alle $a$ gilt), und

б. daß dem Bilde $n^{\prime}$ jedes solchen Elementes $n$ von $A_{0}$, welches

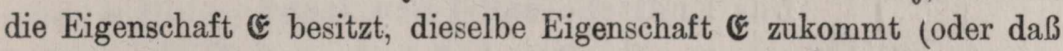
der Satz $\mathfrak{S}$, sobald er für ein Element $n$ von $A_{0}$ gilt, gewiß auch für dessen Bild $n^{\prime}$ gelten muß).

In der Tat, bezeichnet man mit $\Sigma$ das System aller Dinge, welche die Eigenschaft $\mathbb{E}$ besitzen (oder für welche der Satz $\mathfrak{S}$ gilt), so leuchtet die vollständige Übereinstimmung der jetzigen Ausdrucksweise des Satzes mit der in 59 gebrauchten unmittelbar ein.

61. Satz. Die Kette von $\mathfrak{M}(A, B, C \ldots)$ ist $\mathfrak{M}\left(A_{0}, B_{0}, C_{0} \ldots\right)$.

Beweis. Bezeichnet man mit $\boldsymbol{M}$ das erstere, mit $K$ das letztere System, so ist $K$ nach 42 eine Kette. Da nun jedes der Systeme 
$A, B, C \ldots$ nach 45 Teil von einem der Systeme $A_{0}, B_{0}, C_{0} \ldots$, mithin (nach 12) $M 3 K$ ist, so folgt nach 47 auch

$$
M_{0} 3 K \text {. }
$$

Andererseits, da nach 9 jedes der Systeme $A, B, C \ldots$ Teil von $M$, also nach 45,7 auch Teil der Kette $M_{0}$ ist, so muß nach 47 auch jedes der Systeme $A_{0}, B_{0}, C_{0} \ldots$ Teil von $M_{0}$, mithin nach 10

\section{K $3 M_{0}$}

sein, woraus in Verbindung mit dem Obigen der zu beweisende Satz $M_{0}=K$ folgt (5).

62. Satz. Die Kette von $\mathfrak{G}(A, B, C \ldots)$ ist Teil von $\mathfrak{G}\left(A_{0}, B_{0}, C_{0} \ldots\right)$.

Beweis. Bezeichnet man mit $G$ das erstere, mit $K$ das letztere System, so ist $K$ nach 43 eine Kette. Da nun jedes der Systeme $A_{0}, B_{0}, C_{0} \ldots$ nach 45 Ganzes von einem der Systeme $A, B, C \ldots$, mithin (nach 20) $G 3 K$ ist, so folgt aus 47 der zu beweisende Satz $G_{0} 3 K$.

63. Satz. Ist $K^{\prime} 3 L 3 K$, also $K$ eine Kette, so ist auch $L$ eine Kette. Ist dieselbe echter Teil von $K$, und $U$ das System aller derjenigen Elemente von $K$, die nicht in $L$ enthalten sind, ist ferner die Kette $U_{0}$ echter Teil von $K$, und $V$ das System aller derjenigen Elemente von $K$, die nicht in $U_{0}$ enthalten sind, so ist $K=\mathfrak{M}\left(U_{0}, V\right)$ und $L=\mathfrak{M}\left(U_{0}^{\prime}, V\right)$. Ist endlich $L=K^{\prime}$, so ist $V 3 V^{\prime}$.

Der Beweis dieses Satzes, von dem wir (wie von den beiden vorhergehenden) keinen Gebrauch machen werden, möge dem Leser überlassen bleiben.

\section{$\S 5$.}

Das Endliche und Unendliche.

64. Erklärung*). Ein System $S$ heißt unendlich, wenn es einem echten Teile seiner selbst ähnlich ist (32); im entgegengesetzten Falle heißt $S$ ein endliches System.

*) Will man den Begriff ähnlicher Systeme (32) nicht benutzen, so muß man sagen: $S$ heißt unendlich, wenn es einen echten Teil von $S$ gibt (6), in welchem $S$ sich deutlich (ähnlich) abbilden läßt $(26,36)$. In dieser Form habe ich die Definition des Unendlichen, welche den Kern meiner ganzen Untersuchung bildet, im September 1882 Herrn G. Cantor und schon mehrere Jahre früher auch den Herren Schwarz und Weber mitgeteilt. Alle anderen mir bekannten Versuche, das Unendliche vom Endlichen zu unterscheiden, scheinen mir so wenig gelungen zu sein, daß ich auf eine Kritik derselben verzichten zu dürfen glaube. 
65. Satz. Jedes aus einem einzigen Elemente bestehende System ist endlich.

Beweis. Denn ein solches System besitzt gar keinen echten Teil $(2,6)$.

66. Satz. Es gibt unendliche Systeme.

Beweis*). Meine Gedankenwelt, d. h. die Gesamtheit $S$ aller Dinge, welche Gegenstand meines Denkens sein können, ist unendlich. Denn wenn $s$ ein Element von $S$ bedeutet, so ist der Gedanke $s^{\prime}$, daß $s$ Gegenstand meines Denkens sein kann, selbst ein Element von $S$. Sieht man dasselbe als Bild $\varphi(s)$ des Elementes $s$ an, so hat daher die hierdurch bestimmte Abbildung $\varphi$ von $S$ die Eigenschaft, daß das Bild $S^{\prime}$ Teil von $S$ ist; und zwar ist $S^{\prime}$ echter Teil von $S$, weil es in $S$ Elemente gibt (z. B. mein eigenes Ich), welche von jedem solchen Gedanken $s^{\prime}$ verschieden und deshalb nicht in $S^{\prime}$ enthalten sind. Endlich leuchtet ein, daß, wenn $a, b$ verschiedene Elemente von $S$ sind, auch ihre Bilder $a^{\prime}, b^{\prime}$ verschieden sind, daß also die Abbildung $\varphi$ eine deutliche (ähnliche) ist (26). Mithin ist $S$ unendlich, w. z. b. w.

67. Satz. Sind $R, S$ ähnliche Systeme, so ist $R$ endlich oder unendlich, je nachdem $S$ endlich oder unendlich ist.

Beweis. Ist $S$ unendlich, also ähnlich einem echten Teile $S^{\prime}$ seiner selbst, so muß, wenn $R$ und $S$ ähnlich sind, $S^{\prime}$ nach 33 ähnlich mit $R$ und nach 35 zugleich ähnlich mit einem echten Teile von $R$ sein, welcher mithin nach 33 selbst ähnlich mit $R$ ist; also ist $R$ unendlich, w. z. b. w.

68. Satz. Jedes System $S$, welches einen unendlichen Teil $T$ besitzt, ist ebenfalls unendlich; oder mit anderen Worten, jeder Teil eines endlichen Systems ist endlich.

Beweis. Ist $T$ unendlich, gibt es also eine solche ähnliche Abbildung $\psi$ von $T$, daß $\psi(T)$ ein echter Teil von $T$ wird, so kann man, wenn $T$ Teil von $S$ ist, diese Abbildung $\psi$ zu einer Abbildung $\varphi$ von $S$ erweitern, indem man, wenn $s$ irgendein Element von $S$ bedeutet, $\varphi(s)=\psi(s)$ oder $\varphi(s)=s$ setzt, je nachdem $s$ Element von $T$ ist oder nicht. Diese Abbildung $\varphi$ ist eine ähnliche; bedeuten nämlich $a, b$ verschiedene Elemente von $S$, so ist, wenn sie zugleich

*) Eine ähnliche Betrachtung findet sich in $\S 13$ der Paradoxien des Unendlichen von Bolzano (Leipzig 1851). 
in $T$ enthalten sind, das Bild $\varphi(a)=\psi(a)$ verschieden von dem Bilde $\varphi(b)=\psi(b)$, weil $\psi$ eine ähnliche Abbildung ist; wenn ferner $a$ in $T, b$ nicht in $T$ enthalten ist, so ist $\varphi(a)=\psi(a)$ verschieden von $\varphi(b)=b$, weil $\psi(a)$ in $T$ enthalten ist; wenn endlich weder $a$ noch $b$ in $T$ enthalten ist, so ist ebenfalls $\varphi(a)=a$ verschieden von $\varphi(b)=b$, was zu zeigen war. Da ferner $\psi(T)$ Teil von $T$, also nach 7 auch Teil von $S$ ist, so leuchtet ein, daß auch $\varphi(S) 3 S$ ist. Da endlich $\psi(T)$ echter Teil von $T$ ist, so gibt es in $T$, also auch in $S$ ein Element $t$, welches nicht in $\psi(T)=\varphi(T)$ enthalten ist; da nun das Bild $\varphi(s)$ jedes nicht in $T$ enthaltenen Elementes $s$ selbst $=s$, also auch von $t$ verschieden ist, so kann $t$ überhaupt nicht in $\varphi(S)$ enthalten sein; mithin ist $\varphi(S)$ echter Teil von $S$, und folglich ist $S$ unendlich, w. z. b. w.

69. Satz. Jedes System, welches einem Teile eines endlichen Systems ähnlich ist, ist selbst endlich.

Der Beweis folgt aus 67, 68.

70. Satz. Ist $a$ ein Element von $S$, und ist der Inbegriff $T$ aller von $a$ verschiedenen Elemente von $S$ endlich, so ist auch $S$ endlich.

Beweis. Wir haben (nach 64 ) zu zeigen, daß, wenn $\varphi$ irgendeine ähnliche Abbildung von $S$ in sich selbst bedeutet, das Bild $\varphi(S)$ oder $S^{\prime}$ niemals ein echter Teil von $S$, sondern immer $=S$ ist. Offenbar ist $S=\mathfrak{M}(a, T)$, und folglich nach 23, wenn die Bilder wieder durch Akzente bezeichnet werden, $S^{\prime}=\mathfrak{M}\left(a^{\prime}, T^{\prime}\right)$, und wegen der Ähnlichkeit der Abbildung $\varphi$ ist $a^{\prime}$ nicht in $T^{\prime}$ enthalten (26). Da ferner nach Annahme $S^{\prime} 3 S$ ist, so muß $a^{\prime}$ und ebenso jedes Element von $T^{\prime}$ entweder $=a$ oder Element von $T$ sein. Wenn daher - welchen Fall wir zunächst behandeln wollen - $a$ nicht in $T^{\prime}$ enthalten ist, so muß $T^{\prime} 3 T$ und folglich $T^{\prime}=T$ sein, weil $\varphi$ eine ähnliche Abbildung und weil $T$ ein endliches System ist; und da $a^{\prime}$, wie bemerkt, nicht in $T^{\prime}$, d. h. nicht in $T$ enthalten ist, so $\mathrm{mu} ß$ $a^{\prime}=a$ sein, und folglich ist in diesem Falle wirklich $S^{\prime}=S$, wie behauptet war. Im entgegengesetzten Falle, wenn $a$ in $T^{\prime}$ enthalten und folglich das Bild $b^{\prime}$ eines in $T$ enthaltenen Elementes $b$ ist, wollen wir mit $U$ den Inbegriff aller derjenigen Elemente $u$ von $T$ bezeichnen, welche von $b$ verschieden sind; dann ist $T=\mathfrak{M}(b, U)$ und (nach 15) $S=\mathfrak{M}(a, b, U)$, also $S^{\prime}=\mathfrak{N}\left(a^{\prime}, a, U^{\prime}\right)$. Wir bestimmen nun eine neue Abbildung $\psi$ von $T$, indem wir $\psi(b)=a^{\prime}$ 
und allgemein $\psi(u)=u^{\prime}$ setzen, wodurch (nach 23) $\psi(T)=\mathfrak{M}\left(a^{\prime}, U^{\prime}\right)$ wird. Offenbar ist $\psi$ eine ähnliche Abbildung, weil $\varphi$ eine solche war, und weil $a$ nicht in $U$, also auch $a^{\prime}$ nicht in $U^{\prime}$ enthalten ist. Da ferner $a$ und jedes Element $u$ verschieden von $b$ ist, so muß (wegen der Ähnlichkeit von $\varphi$ ) auch $a^{\prime}$ und jedes Element $u^{\prime}$ verschieden von $a$ und folglich in $T$ enthalten sein; mithin ist $\psi(T) 3 T$, und da $T$ endlich ist, so muß $\psi(T)=T$, also $\mathfrak{M}\left(a^{\prime}, U^{\prime}\right)=T$ sein. Hieraus folgt aber (nach 15)

$$
\mathfrak{M}\left(a^{\prime}, a, U^{\prime}\right)=\mathfrak{M}(a, T),
$$

d. h. nach dem Obigen $S^{\prime}=S$. Also ist auch in diesem Falle der erforderliche Beweis geführt.

\section{$\S 6$.}

Einfach unendliche Systeme. Reihe der natürlichen Zahlen.

71. Erklärung. Ein System $N$ heißt einfach unendlich, wenn es eine solche ähnliche Abbildung $\varphi$ von $N$ in sich selbst gibt, daß $N$ als Kette (44) eines Elementes erscheint, welches nicht in $\varphi(N)$ enthalten ist. Wir nennen dies Element, das wir im folgenden durch das Symbol 1 bezeichnen wollen, das Grundelement von $N$ und sagen zugleich, das einfach unendliche System $N$ sei durch diese Abbildung $\varphi$ geordnet. Behalten wir die früheren bequemen $\mathrm{Be}$ zeichnungen für die Bilder und Ketten bei (§4), so besteht mithin das Wesen eines einfach unendlichen Systems $N$ in der Existenz einer Abbildung $\varphi$ von $N$ und eines Elementes 1, die den folgenden Bedingungen $\alpha, \beta, \gamma, \delta$ genügen:

$$
\begin{array}{ll}
\alpha . & N^{\prime} 3 N . \\
\beta . & N=10^{.} \\
\gamma . & \text { Das Element } 1 \text { ist nicht in } N^{\prime} \text { enthalten. } \\
\delta . & \text { Die Abbildung } \varphi \text { ist ähnlich. }
\end{array}
$$

Offenbar folgt aus $\alpha, \gamma, \delta$, daß jedes einfach unendliche System $N$ wirklich ein unendliches Systern ist (64), weil es einem echten Teile $N^{\prime}$ seiner selbst ähnlich ist.

72. Satz. In jedem unendlichen System $S$ ist ein einfach unendliches System $N$ als Teil enthalten.

Beweis. Es gibt nach 64 eine solche ähnliche Abbildung $\varphi$ von $S$, daß $\varphi(S)$ oder $S^{\prime}$ ein echter Teil von $S$ wird; es gibt also ein Element 1 in $S$, welches nicht in $S^{\prime}$ enthalten ist. Die Kette 
$N=1_{0}$, welche dieser Abbildung $\varphi$ des Systems $S$ in sich selbst entspricht (44), ist ein einfach unendliches, durch $\varphi$ geordnetes System; denn die charakteristischen Bedingungen $\alpha, \beta, \gamma, \delta$ in 71 sind offenbar sämtlich erfüllt.

73. Erklärung. Wenn man bei der Betrachtung eines einfach unendlichen, durch eine Abbildung $\varphi$ geordneten Systems $N$ von der besonderen Beschaffenheit der Elemente gänzlich absieht, lediglich ihre Unterscheidbarkeit festhält und nur die Beziehungen auffaßt, in die sie durch die ordnende Abbildung $\varphi$ zueinander gesetzt sind, so heißen diese Elemente natürliche Zahlen oder Ordinalzahlen oder auch schlechthin Zahlen, und das Grundelement 1 heißt die Grundzahl der Zahlenreihe $N$. In Rücksicht auf diese Befreiung der Elemente von jedem anderen Inhalt (Abstraktion) kann man die Zahlen mit Recht eine freie Schöpfung des menschlichen Geistes nennen. Die Beziehungen oder Gesetze, welche ganz allein aus den Bedingungen $\alpha, \beta, \gamma, \delta$ in 71 abgeleitet werden und deshalb in allen geordneten einfach unendlichen Systemen immer dieselben sind, wie auch die den einzelnen Elementen zufällig gegebenen Namen lauten mögen (vgl. 134), bilden den nächsten Gegenstand der Wissenschaft von den Zahlen oder der Arithmetik. Aus den allgemeinen Begriffen und Sätzen des $\S 4$ über Abbildung eines Systems in sich selbst entnehmen wir zunächst unmittelbar die folgenden Grundsätze, wobei unter $a, b \ldots m, n \ldots$ stets Elemente von $N$, also Zahlen, unter $A, B, C \ldots$ Teile von $N$, unter $a^{\prime}, b^{\prime} \ldots m^{\prime}, n^{\prime} \ldots A^{\prime}, B^{\prime}, C^{\prime} \ldots$ die entsprechenden Bilder verstanden werden, welche durch die ordnende Abbildung $\varphi$ erzeugt und stets wieder Elemente oder Teile von $N$ sind; das Bild $n^{\prime}$ einer Zahl $n$ wird auch die auf $n$ folgende Zahl genainnt.

74. Satz. Jede Zahl $n$ ist nach 45 in ihrer Kette $n_{0}$ enthalten, und nach 53 ist die Bedingung $n 3 m_{0}$ gleichwertig mit $n_{0} 3 m_{0}$.

75. Satz. Zufolge 57 ist $n_{0}^{\prime}=\left(n_{0}\right)^{\prime}=\left(n^{\prime}\right)_{0}$.

76. Satz. Zufolge 46 ist $n_{0}^{\prime} 3 n_{0}$.

77. Satz. Zufolge 58 ist $n_{0}=\mathfrak{M}\left(n, n_{0}^{\prime}\right)$.

78. Satz. Es ist $N=\mathfrak{M}\left(1, N^{\prime}\right)$, also ist jede von der Grundzahl 1 verschiedene Zahl Element von $N^{\prime}$, d. h. Bild einer Zahl.

Der Beweis folgt aus 77 und 71 .

79. Satz. $N$ ist die einzige Zahlenkette, in welcher die Grundzahl 1 enthalten ist. 
Beweis. Denn wenn 1 Element einer Zahlenkette $K$ ist, so ist nach 47 die zugehörige Kette $N 3 K$, folglich $N=K$, weil selbstverständlich $K 3 N$ ist.

80. Satz der vollständigen Induktion (Schluß von $n$ auf $n^{\prime}$ ). Um zu beweisen, daß ein Satz für alle Zahlen $n$ einer Kette $m_{0}$ gilt, genügt es zu zeigen,

œ. daß er für $n=m$ gilt, und

б. daß aus der Gültigkeit des Satzes für eine Zahl $n$ der Kette $m_{0}$ stets seine Gültigkeit auch für die folgende Zahl $n^{\prime}$ folgt.

Dies ergibt sich unmittelbar aus dem allgemeineren Satze 59 oder 60. Am häufigsten wird der Fall auftreten, wo $m=1$, also $m_{0}$ die volle Zahlenreihe $N$ ist.

\section{$\S 7$.}

Größere und kleinere Zahlen.

81. Satz. Jede Zahl $n$ ist verschieden von der auf sie folgenden Zahl $n^{\prime}$.

Beweis durch vollständige Induktion (80). Denn

@. der Satz ist wahr für die Zahl $n=1$, weil sie nicht in $N^{\prime}$ enthalten ist (71), während die folgende Zahl $1^{\prime}$ als Bild der in $N$ enthaltenen Zahl 1 Element von $N^{\prime}$ ist.

б. Ist der Satz wahr für eine Zahl $n$, und setzt man die folgende Zahl $n^{\prime}=p$, so ist $n$ verschieden von $p$, woraus nach 26 wegen der Ähnlichkeit (71) der ordnenden Abbildung $\varphi$ folgt, daß $n^{\prime}$, also $p$ verschieden von $p^{\prime}$ ist. Mithin gilt der Satz auch für die auf $n$ folgende Zahl $p$, w. z. b. w.

82. Satz. In der Bildkette $n_{0}^{\prime}$ einer Zahl $n$ ist zwar (nach 74,75 ) deren Bild $n^{\prime}$, nicht aber die Zahl $n$ selbst enthalten.

Beweis durch vollständige Induktion (80). Denn

o. der Satz ist wahr für $n=1$, weil $1_{0}^{\prime}=N^{\prime}$, und weil nach 71 die Grundzahl 1 nicht in $N^{\prime}$ enthalten ist.

б. Ist der Satz wahr für eine Zahl $n$, und setzt man wieder $n^{\prime}=p$, so ist $n$ nicht in $p_{0}$ enthalten, also verschieden von jeder in $p_{0}$ enthaltenen Zahl $q$, woraus wegen der Ähnlichkeit von $\varphi$ folgt, daß $n^{\prime}$, also $p$ verschieden von jeder in $p_{0}^{\prime}$ enthaltenen Zahl $q^{\prime}$, also nicht in $p_{0}^{\prime}$ enthalten ist. Mithin gilt der Satz auch für die auf $n$ folgende Zahl $p$, w. z. b. w. 
83. Satz. Die Bildkette $n_{0}^{\prime}$ ist echter Teil der Kette $n_{0}$.

Der Beweis folgt aus $76,74,82$.

84. Satz. Aus $m_{0}=n_{0}$ folgt $m=n$.

Beweis. Da (nach 74) $m$ in $m_{0}$ enthalten, und

$$
m_{0}=n_{0}=\mathfrak{M}\left(n, n_{0}^{\prime}\right)
$$

ist (77), so müßte, wenn der Satz falsch, also $m$ verschieden von $n$ wäre, $m$ in der Kette $n_{0}^{\prime}$ enthalten, folglich nach 74 auch $m_{0} 3 n_{0}^{\prime}$, d. h. $n_{0} 3 n_{0}^{\prime}$ sein; da dies dem Satze 83 widerspricht, so ist unser Satz bewiesen.

85. Satz. Wenn die Zahl $n$ nicht in der Zahlenkette $K$ enthalten ist, so ist $K 3 n_{0}^{\prime}$.

Beweis durch vollständige Induktion (80). Denn

@. der Satz ist nach 78 wahr für $n=1$.

б. Ist der Satz wahr für eine Zahl $n$, so gilt er auch für die folgende Zahl $p=n^{\prime}$; denn wenn $p$ in der Zahlenkette $K$ nicht enthalten ist, so kann nach 40 auch $n$ nicht in $K$ enthalten sein, und folglich ist nach unserer Annahme $K 3 n_{0}^{\prime}$; da nun (nach 77) $\boldsymbol{n}_{0}^{\prime}=p_{0}=\mathfrak{M}\left(p, p_{0}^{\prime}\right)$, also $K 3 \mathfrak{M}\left(p, p_{0}^{\prime}\right)$, und $p$ nicht in $K$ enthalten ist, so muß $K 3 p_{0}^{\prime}$ sein, w. z. b. w.

86. Satz. Wenn die Zahl $n$ nicht in der Zahlenkette $K$ enthalten ist, wohl aber ihr Bild $n^{\prime}$, so ist $K=n_{0}^{\prime}$.

Beweis. Da $n$ nicht in $K$ enthalten ist, so ist (nach 85) $K 3 n_{0}^{\prime}$, und da $n^{\prime} 3 K$, so ist nach 47 auch $n_{0}^{\prime} 3 K$, folglich $K=n_{0}^{\prime}$, w. z. b. w.

87. Satz. In jeder Zahlenkette $K$ gibt es eine und (nach 84) nur eine Zahl $k$, deren Kette $k_{0}=K$ ist.

Beweis. Ist die Grundzahl 1 in $K$ enthalten, so ist (nach 79) $K=N=1_{0}$. Im entgegengesetzten Falle sei $Z$ das System aller nicht in $K$ enthaltenen Zahlen; da die Grundzahl 1 in $Z$ enthalten, aber $Z$ nur ein echter Teil der Zahlenreihe $N$ ist, so kann (nach 79) $Z$ keine Kette, d. h. $Z^{\prime}$ kann nicht Teil von $Z$ sein; es gibt daher in $Z$ eine Zahl $n$, deren Bild $n^{\prime}$ nicht in $Z$, also gewiß in $K$ enthalten ist; da ferner $n$ in $Z$, also nicht in $K$ enthalten ist, so ist (nach 86) $K=n_{0}^{\prime}$, also $k=n^{\prime}$, w. z. b.w.

88. Satz. Sind $m, n$ verschiedene Zahlen, so ist eine und (nach 83, 84) nur eine der Ketten $m_{0}, n_{0}$ echter Teil der anderen, und zwar ist entweder $n_{0} 3 m_{0}^{\prime}$ oder $m_{0} 3 n_{0}^{\prime}$. 
Beweis. Ist $n$ in $m_{0}$ enthalten, also nach 74 auch $n_{0} 3 m_{0}$, so kann $m$ nicht in der Kette $n_{0}$ enthalten sein (weil sonst nach 74 auch $m_{0} 3 n_{0}$, also $m_{0}=n_{0}$, mithin nach 84 auch $m=n$ wäre), and hieraus folgt nach 85 , daß $n_{0} 3 m_{0}^{\prime}$ ist. Im entgegengesetzten Falle, wenn $n$ nicht in der Kette $m_{0}$ enthalten ist, muß (nach 85) $m_{0} 3 n_{0}^{\prime}$ sein, w. z. b.w.

89. Erklärung. Die Zahl $m$ heißt kleiner als die Zahl $n$, und zugleich heißt $n$ größer als $m$, in Zeichen

wenn die Bedingung

$$
m<n \text { und } n>m \text {, }
$$

$$
n_{0} 3 m_{0}^{\prime}
$$

erfüllt ist, welche nach 74 auch durch

ausgedrückt werden kann.

$$
n 3 m_{0}^{\prime}
$$

90. Satz. Sind $m, n$ irgendwelche Zahlen, so findet immer einer und nur einer der folgenden Fälle $\lambda, \mu, v$ statt:

$$
\begin{array}{ll}
\text { A. } & m=n, \quad n=m, \text { d. h. } m_{0}=n_{0}, \\
\mu . & m<n, \quad n>m, \text { d.h. } n_{0} 3 m_{0}^{\prime}, \\
\nu . & m>n, \quad n<m, \text { d. h. } m_{0} 3 n_{0}^{\prime} .
\end{array}
$$

Beweis. Denn wenn $\lambda$ stattfindet (84), so kann weder $\mu$ noch $\nu$ eintreten, weil nach 83 niemals $n_{0} 3 n_{0}^{\prime}$ ist. Wenn aber $\lambda$ nicht stattfindet, so tritt nach 88 einer und nur einer der Fälle $\mu, \nu$ ein, w. z. b.w.

91. Satz. Es ist $n<n^{\prime}$.

Beweis. Denn die Bedingung für den Fall $v$ in 90 wird durch $m=n^{\prime}$ erfüllt.

92. Erklärung. Um auszudrücken, daß $m$ entweder $=n$ oder $<n$, also nicht $>n$ ist (90), bedient man sich der Bezeichnung

$$
m \leqq n \text { oder auch } n \geqq m,
$$

und man sagt, $m$ sei höchstens gleich $n$, und $n$ sei mindestens gleich $m$.

93. Satz. Jede der Bedingungen

$$
m \leqq n, \quad m<n^{\prime}, \quad n_{0} 3 m_{0}
$$

ist gleichwertig mit jeder der anderen.

Beweis. Denn wenn $m \leqq n$, so folgt aus $\lambda, \mu$ in 90 immer $n_{0} 3 m_{0}$, weil (nach 76) $m_{0}^{\prime} 3 m_{0}$ ist. Umgekehrt, wenn $n_{0} 3 m_{0}$, also 
nach 74 auch $n 3 m_{0}$ ist, so folgt aus $m_{0}=\mathfrak{M}\left(m, m_{0}^{\prime}\right)$, daß entweder $n=m$ oder $n 3 m_{0}^{\prime}$, d. h. $n>m$ ist. Mithin ist die Bedingung $m \leqq n$ gleichwertig mit $n_{0} 3 m_{0}$. Außerdem folgt aus 22, 27, 75, daß diese Bedingung $n_{0} 3 m_{0}$ wieder gleichwertig mit $n_{0}^{\prime} 3 m_{0}^{\prime}$, d. h. (nach $\mu$ in 90) mit $m<n^{\prime}$ ist, w. z. b.w.

94. Satz. Jede der Bedingungen

$$
m^{\prime} \leqq n, \quad m^{\prime}<n^{\prime}, \quad m<n
$$

ist gleichwertig mit jeder der anderen.

Der Beweis folgt unmittelbar aus 93, wenn man dort $m$ durch $m^{\prime}$ ersetzt, und aus $\mu$ in 90 .

95. Satz. Wenn $l<m$ und $m \leqq n$, oder wenn $l \leqq m$ und $m<n$, so ist $l<n$. Wenn aber $l \leqq m$ und $m \leqq n$, so ist $l \leqq n$.

Beweis. Denn aus den (nach 89, 93) entsprechenden Bedingungen $m_{0} 3 l_{0}^{\prime}$ und $n_{0} 3 m_{0}$ folgt (nach 7) $n_{0} 3 l_{0}^{\prime}$, und dasselbe folgt auch aus den Bedingungen $m_{0} 3 l_{0}$ und $n_{0} 3 m_{0}^{\prime}$, weil zufolge der ersteren auch $m_{0}^{\prime} 3 l_{0}^{\prime}$ ist. Endlich folgt aus $m_{0} 3 l_{0}$, und $n_{0} 3 m_{0}$ auch $n_{0} 3 l_{0}$, w. z. b.w.

96. Satz. In jedem Teile $T$ von $N$ gibt es eine und nur eine kleinste Zahl $k$, d.h. eine Zahl $k$, welche kleiner ist als jede andere in $T$ enthaltene Zahl. Besteht $T$ aus einer einzigen Zahl, so ist dieselbe auch die kleinste Zahl in $T$.

Beweis. Da $T_{0}$ eine Kette ist (44), so gibt es nach 87 eine Zahl $k$, deren Kette $k_{0}=T_{0}$ ist. $\mathrm{Da}$ hieraus (nach 45,77 ) $T 3 \mathfrak{M}\left(k, k_{0}^{\prime}\right)$ folgt, so $\mathrm{mu}$ zunächst $k$ selbst in $T$ enthalten sein (weil sonst $T 3_{0}^{\mathrm{z}} k_{0}^{\prime}$, also nach 47 auch $T_{0} 3 k_{0}^{n}$, d.h. $k_{0} 3 k_{0}^{\prime}$ wäre, was nach 83 unmöglich ist), und außerdem muß jede von $k$ verschiedene Zahl des Systems $T$ in $k_{0}^{\prime}$ enthalten, d.h. $>\boldsymbol{k}$ sein (89), woraus zugleich nach 90 folgt, daß es nur eine einzige kleinste Zahl in $T$ gibt, w. z. b.w.

97. Satz. Die kleinste Zahl der Kette $n_{0}$ ist $n$, und die Grundzahl 1 ist die kleinste aller Zahlen.

Beweis. Denn nach 74, 93 ist die Bedingung $m 3 n_{0}$ gleichwertig mit $m \geqq n$. Oder es folgt unser Satz auch unmittelbar aus dem Beweise des vorhergehenden Satzes, weil, wenn daselbst $T=n_{0}$ angenommen wird, offenbar $k=n$ wird (51). 
98. Erklärung. Ist $n$ irgendeine Zahl, so wollen wir mit $Z_{n}$ das System aller Zahlen bezeichnen, welche nicht größer als $n$, also nicht in $n_{0}^{\prime}$ enthalten sind. Die Bedingung

$$
m 3 Z_{n}
$$

ist nach 92, 93 offenbar gleichwertig mit jeder der folgenden Bedingungen:

$$
m \leqq n, \quad m<n^{\prime}, \quad n_{0} 3 m_{0} .
$$

99. Satz. Es ist $13 Z_{n}$ und $n 3 Z_{n}$.

Der Beweis folgt aus 98 oder auch aus 71 und 82 .

100. Satz. Jede der nach 98 gleichwertigen Bedingungen

$$
m 3 Z_{n}, \quad m \leqq n, \quad m<n^{\prime}, \quad n_{0} 3 m_{0}
$$

ist auch gleichwertig mit der Bedingung

$$
Z_{m} 3 Z_{n} \text {. }
$$

Beweis. Denn wenn $m 3 Z_{n}$, also $m \leqq n$, und wenn $l 3 Z_{m}$, also $l \leqq m$, so ist nach 95 auch $l \leqq n$, d. h. $l 3 Z_{n}$; wenn also $m 3 Z_{n}$, so ist jedes Element $l$ des Systems $Z_{m}$ auch Element von $\boldsymbol{Z}_{n}$, d.h. $Z_{m} 3 Z_{n}$. Umgekehrt, wenn $Z_{m} 3 Z_{n}$, so muß nach 7 auch $m 3 Z_{n}$ sein, weil (nach 99) $m 3 Z_{m}$ ist, w. z. b.w.

101. Satz. Die Bedingungen für die Fälle $\lambda, \mu, \nu$ in 90 lassen sich auch in folgender Weise darstellen:

$$
\begin{array}{ll}
\text { ג. } & m=n, \quad n=m, \quad Z_{m}=Z_{n}, \\
\mu . & m<n, \quad n>m, \quad Z_{m^{\prime}} 3 Z_{n}, \\
\nu . & m>n, \quad n<m,
\end{array}
$$

Der Beweis folgt unmittelbar aus 90 , wenn man bedenkt, daß nach 100 die Bedingungen $n_{0} 3 m_{0}$ und $Z_{m} 3 Z_{n}$ gleichwertig sind.

102. Satz. Es ist $Z_{1}=1$.

Beweis. Denn die Grundzahl 1 ist nach 99 in $Z_{1}$ enthalten, und jede von 1 verschiedene $\mathrm{Zahl}$ ist nach 78 in $1_{0}^{\prime}$, also nach 98 nicht in $Z_{1}$ enthalten, w. z. b.w.

103. Satz. Zufolge 98 ist $N=\mathfrak{M}\left(Z_{n}, n_{0}^{\prime}\right)$.

104. Satz. Es ist $n=\mathfrak{G}\left(Z_{n}, n_{0}\right)$, d. h. $n$ ist das einzige emeinsame Element der Systeme $Z_{n}$ und $n_{0}$.

Beweis. Aus 99 und 74 folgt, daß $n$ in $Z_{n}$ und $n_{0}$ enthalten ist; aber jedes von $n$ verschiedene Element der Kette $n_{0}$ ist nach 77 in $n_{0}^{\prime}$, also nach 98 nicht in $Z_{n}$ enthalten, w. z. b.w.

105. Satz. Zufolge 91,98 ist die $Z$ ahl $n^{\prime}$ nicht in $Z_{n}$ enthalten. 
106. Satz. Ist $m<n$, so ist $Z_{m}$ echter Teil von $Z_{n}$, und umgekehrt.

Beweis. Wenn $m<n$, so ist (nach 100) $Z_{m} 3 Z_{n}$, und da die nach 99 in $Z_{n}$ enthaltene Zahl $n$ nach 98 nicht in $Z_{m}$ enthalten sein kann, weil $n>m$ ist, so ist $Z_{m}$ echter Teil von $Z_{n}$. Umgekehrt, wenn $Z_{m}$ echter Teil von $Z_{n}$, so ist (nach 100) $m \leqq n$, und da $m$ nicht $=n$ sein kann, weil sonst auch $Z_{m}=Z_{n}$ wäre, so $m u ß m<n$ sein, w. z. b. w.

107. Satz. $Z_{n}$ ist echter Teil von $Z_{n^{\prime}}$.

Der Beweis folgt aus 106, weil (nach 91) $n<n^{\prime}$ ist.

108. Satz. $Z_{n^{\prime}}=\mathfrak{M}\left(Z_{n}, n^{\prime}\right)$.

Beweis. Denn jede in $Z_{n^{\prime}}$ enthaltene Zahl ist (nach 98) $\leqq n^{\prime}$, also entweder $=n^{\prime}$, oder $<n^{\prime}$ und folglich nach 98 Element von $Z_{n}$; mithin ist gewiß $Z_{n^{\prime}} 3 \mathfrak{M}\left(Z_{n}, n^{\prime}\right)$. Da umgekehrt (nach 107) $Z_{n} 3 Z_{n^{\prime}}$ und (nach 99) $n^{\prime} 3 Z_{n^{\prime}}$ ist, so folgt (nach 10)

$$
\mathfrak{M}\left(Z_{n}, n^{\prime}\right) 3 Z_{n^{\prime}}
$$

woraus sich unser Satz nach 5 ergibt.

109. Satz. Das Bild $Z_{n}^{\prime}$ des Systems $Z_{n}$ ist echter Teil des Systems $Z_{n^{\prime}}$.

Beweis. Denn jede in $Z_{n}^{\prime}$ enthaltene Zahl ist das Bild $m^{\prime}$ einer in $Z_{n}$ enthaltenen Zahl $m$, und da $m \leqq n$, also (nach 94) $m^{\prime} \leqq n^{\prime}$, so folgt (nach 98) $Z_{n}^{\prime} 3 Z_{n^{\prime}}$. Da ferner die Zahl 1 nach 99 in $Z_{n^{\prime}}$, aber nach 71 nicht in dem Bilde $Z_{n}^{\prime}$ enthalten sein kann, so ist $Z_{n}^{\prime}$ echter Teil von $Z_{n^{\prime}}$, w. z. b.w.

110. Satz. $Z_{n^{\prime}}=\mathfrak{M i}\left(1, Z_{n}^{\prime}\right)$.

Beweis. Jede von 1 verschiedene $Z$ Zahl des Systems $Z_{n^{\prime}}$ ist nach 78 das Bild $m^{\prime}$ einer Zahl $m$, und diese muß $\leqq n$, also nach 98 in $Z_{n}$ enthalten sein (weil sonst $m>n$, also nach 94 auch $m^{\prime}>n^{\prime}$, mithin $m^{\prime}$ nach 98 nicht in $Z_{n^{\prime}}$ enthalten wäre); aus $m 3 Z_{n}$ folgt aber $m^{\prime} 3 Z_{n}^{\prime}$, und folglich ist gewiß

$$
Z_{n^{\prime}} 3 \mathfrak{M}\left(1, Z_{n}^{\prime}\right) \text {. }
$$

Da umgekehrt (nach 99) $13 Z_{n^{\prime}}$ und (nach 109) $Z_{n}^{\prime} 3 Z_{n^{\prime}}$, so folgt (nach 10) $\mathfrak{M}\left(1, Z_{n}^{\prime}\right) 3 Z_{n^{\prime}}$, und hieraus ergibt sich unser Satz nach 5 .

111. Erklärung. Wenn es in einem System $E$ von Zahlen ein Element $g$ gibt, welches größer als jede andere in $E$ enthaltene Zahl ist, so heißt $g$ die größte Zahl des Systems $\boldsymbol{E}$, und offenbar kann es nach 90 nur eine solche größte Zahl in $E$ geben. Besteht 
ein System aus einer einzigen Zahl, so ist diese selbst die größte Zahl des Systems.

112. Satz. Zufolge 98 ist $n$ die größte Zahl des Systems $Z_{n}$.

113. Satz. Gibt es in $E$ eine größte $Z a h l ~ g$, so ist $E 3 Z_{g}$.

Beweis. Denn jede in $E$ enthaltene Zahl ist $\leqq g$, mithin nach 98 in $Z_{g}$ enthalten, w. z. b. w.

114. Satz. Ist $E$ Teil eines Systems $Z_{n}$, oder gibt es, was dasselbe sagt, eine Zahl $n$ von der Art, daß alle in $E$ enthaltenen Zahlen $\leqq n$ sind, so besitzt $E$ eine größte Zahl $g$.

Beweis. Das System aller Zahlen $p$, welche der Bedingung $E 3 Z_{p}$ genügen - und nach unserer Annahme gibt es solche -, ist eine Kette (37), weil nach 107, 7 auch $E 3 Z_{p^{\prime}}$ folgt, und ist daher (nach 87$)=g_{0}$, wo $g$ die kleinste dieser Zahlen bedeutet $(96,97)$. Es ist daher auch $E 3 Z_{g}$, folglich (98) ist jede in $E$ enthaltene Zahl $\leqq g$, und wir haben, nur noch zu zeigen, daß die Zahl $g$ selbst in $E$ enthalten ist. Dies leuchtet unmittelbar ein, wenn $g=1$ ist, weil dann (nach 102) $Z_{g}$ und folglich auch $E$ aus der einzigen Zahl 1 besteht. Ist aber $g$ von 1 verschieden und folglich nach 78 das Bild $f^{\prime}$ einer Zahl $f$, so ist (nach 108) $E 3 \mathfrak{M}\left(Z_{f}, g\right)$; wäre nun $g$ nicht in $E$ enthalten, so müßte $E 3 Z_{f}$ sein, und es gäbe daher unter den Zahlen $p$ eine Zahl $f$, welche (nach 91) $<g$ ist, was dem Obigen widerspricht; mithin ist $g$ in $E$ enthalten, w. z. b.w.

115. Erklärung. Ist $l<m$ und $m<n$, so sagen wir, die Zahl $m$ liege zwischen $l$ und $n$ (auch zwischen $n$ and $l$ ).

116. Satz. Es gibt keine Zahl, die zwischen $n$ und $n^{\prime}$ liegt.

Beweis. Denn sobald $m<n^{\prime}$, also (nach 93) $m \leqq n$ ist, so kann nach 90 nicht $n<m$ sein, w. z. b.w.

117. Satz. Ist $t$ eine Zahl in $T$, aber nicht die kleinste (96), so gibt es in $T$ eine und nur eine nächst kleinere Zahl $s$, d.h. eine Zahl $s$ von der Art, daß $s<t$, und daß es in $T$ keine zwischen $s$ und $t$ liegende Zahl gibt. Ebenso gibt es, wenn nicht etwa $t$ die größte $\mathrm{Zahl}$ in $T$ ist (111), in $T$ immer eine und nur eine nächst größere Zahl $u$, d. h. eine Zahl $u$ von der Art, daß $t<u$, und daß es in $T$ keine zwischen $t$ und $u$ liegende Zahl gibt. Zugleich ist $t$ in $T$ nächst größer als $s$ und nächst kleiner als $u$. Beweis. Wenn $t$ nicht die kleinste Zahl in $T$ ist, so sei $E$ das System aller derjenigen Zahlen von $T$, welche $<\boldsymbol{t}$ sind; dann 
ist (nach 98) $E 3 Z_{t}$, und folglich (114) gibt es in $E$ eine größte Zahl $s$, welche offenbar die im Satze angegebenen Eigenschaften besitzt und auch die einzige solche Zahl ist. Wenn ferner $t$ nicht die größte Zahl in $T$ ist, so gibt es nach 96 unter allen den Zahlen von $T$, welche $>t$ sind, gewiß eine kleinste $u$, welche, und zwar allein, die im Satze angegebenen Eigenschaften besitzt. Ebenso leuchtet die Richtigkeit der Schlußbemerkung des Satzes ein.

118. Satz. In $N$ ist die Zahl $n^{\prime}$ nächst größer als $n$, und $n$ nächst kleiner als $n^{\prime}$.

Der Beweis folgt aus 116, 117.

$\S 8$.

Endliche und unendliche Teile der Zahlenreihe.

119. Satz. Jedes System $Z_{n}$ in 98 ist endlich.

Beweis durch vollständige Induktion (80). Denn

@. der Satz ist wahr für $n=1$ zufolge $65,102$.

б. Ist $Z_{n}$ endlich, so folgt aus 108 und 70 , daß auch $Z_{n^{\prime}}$ endlich ist, w. z. b.w.

120. Satz. Sind $m, n$ verschiedene Zahlen, so sind $Z_{m}, Z_{n}$ unähnliche Systeme.

Beweis. Der Symmetrie wegen dürfen wir nach 90 annehmen, es sei $m<n$; dann ist $Z_{m}$ nach 106 echter Teil von $Z_{n}$, und da $Z_{n}$ nach 119 endlich ist, so können (nach 64) $Z_{m}$ und $Z_{n}$ nicht ähnlich sein, w.z.b.w.

121. Satz. Jeder Teil $E$ der Zahlenreihe $N$, welcher eine größte Zahl besitzt (111), ist endlich.

Der Beweis folgt aus 113, 119, 68.

122. Satz. Jeder Teil $U$ der Zahlenreihe $N$, welcher keine größte Zahl besitzt, ist einfach unendlich (71).

Beweis. Ist $u$ irgendeine $\mathrm{Zahl}$ in $U$, so gibt es nach 117 in $U$ eine und nur eine nächst größere Zahl als $u$, die wir mit $\psi(u)$ bezeichnen und als Bild von $u$ ansehen wollen. Die hierdurch vollständig bestimmte Abbildung $\psi$ des Systems $U$ hat offenbar die Eigenschaft

$$
\text { a. } \psi(U) 3 U \text {, }
$$

d.h. $U$ wird durch $\psi$ in sich selbst abgebildet. Sind ferner $u, v$ verschiedene Zahlen in $U$, so dürfen wir der Symmetrie wegen nach 
90 annehmen, es sei $u<v$; dann folgt nach 117 aus der Definition von $\psi$, daß $\psi(u) \leqq v$ und $v<\psi(v)$, also (nach 95) $\psi(u)<\psi(v)$ ist; mithin sind nach 90 die Bilder $\psi(u), \psi(v)$ verschieden, d.h.

\section{$\delta$. die Abbildung $\psi$ ist ähnlich.}

Bedeutet ferner $u_{1}$ die kleinste Zahl (96) ḋes Systems $U$, so ist jede in $U$ enthaltene Zahl $u \geqq u_{1}$, und da allgemein $u<\psi(u)$, so ist (nach 95) $u_{1}<\psi(u)$, also ist $u_{1}$ nach 90 verschieden von $\psi(u)$, d. h.

$\gamma$. das Element $u_{1}$ von $U$ ist nicht in $\psi(U)$ enthalten.

Mithin ist $\psi(U)$ ein echter Teil von $U$, und folglich ist $U$ nach 64 ein unendliches System. Bezeichnen wir nun in Übereinstimmung mit 44 , wenn $V$ irgendein Teil von $U$ ist, mit $\psi_{0}(V)$ die der Abbildung $\psi$ entsprechende Kette von $V$, so wollen wir endlich noch zeigen, daß

$$
\text { } \beta . U=\psi_{0}\left(u_{1}\right)
$$

ist. In der Tat, da jede solche Kette $\psi_{0}(V)$ zufolge ihrer Definition (44) ein Teil des durch $\psi$ in sich selbst abgebildeten Systems $U$ ist, so ist selbstverständlich $\psi_{0}\left(u_{1}\right) 3 U$; umgekehrt leuchtet aus 45 zunächst ein, daß das in $U$ enthaltene Element $u_{1}$ gewiß in $\psi_{0}\left(u_{1}\right)$ enthalten ist; nehmen wir aber an, es gäbe Elemente von $U$, die nicht in $\psi_{0}\left(u_{1}\right)$ enthalten sind, so muß es unter ihnen nach 96 eine kleinste Zahl $w$ geben, und da dieselbe nach dem eben Gesagten verschieden von der kleinsten Zahl $u_{1}$ des Systems $U$ ist, so muß es nach 117 in $U$ auch eine Zahl $v$ geben, welche nächst kleiner als $w$ ist, woraus zugleich folgt, daß $w=\psi(v)$ ist; da nun $v<w$, so muß $v$ zufolge der Definition von $w$ gewiß in $\psi_{0}\left(u_{1}\right)$ enthalten sein; hieraus folgt aber nach 55 , daß auch $\psi(v)$, also $w$ in $\psi_{0}\left(u_{1}\right)$ enthalten sein muß, und da dies im Widerspruch mit der Definition von $w$ steht, so ist unsere obige Annahme unzulässig; mithin ist U3 $\psi_{0}\left(u_{1}\right)$ und folglich auch $U=\psi_{0}\left(u_{1}\right)$, wie behauptet war. Aus $\alpha, \beta, \gamma, \delta$ geht nun nach 71 hervor, daß $U$ ein durch $\psi$ geordnetes einfach unendliches System ist, w. z. b. w.

123. Satz. Zufolge 121,122 ist irgendein Teil $T$ der Zahlenreihe $N$ endlich oder einfach unendlich, je nachdem es in $T$ eine größte Zahl gibt oder nicht gibt. 
$\S 9$.

Definition einer Abbildung der Zahlenreihe durch Induktion.

124. Wir bezeichnen auch im folgenden mit kleinen lateinischen Buchstaben Zahlen und behalten überhaupt alle Bezeichnungen der vorhergehenden $\S 6$ bis 8 bei, während $\Omega$ ein beliebiges System bedeutet, dessen Elemente nicht notwendig in $N$ enthalten zu sein brauchen.

125. Satz. Ist eine beliebige (ähnliche oder unähnliche) Abbildung $\theta$ eines Systems $\Omega$ in sich selbst, und außerdem ein bestimmtes Element $\omega$ in $\Omega$ gegeben, so entspricht jeder Zahl $n$ eine und nur eine Abbildung $\psi_{n}$ des zugehörigen, in 98 erklärten Zahlensystems $Z_{n}$, welche den Bedingungen*)

I. $\psi_{n}\left(Z_{n}\right) 3 \Omega$,

II. $\psi_{n}(1)=\omega$,

III. $\psi_{n}\left(t^{\prime}\right)=\theta \psi_{n}(t)$, wenn $t<n$, genügt, wo das Zeichen $\theta \psi_{n}$ die in 25 angegebene Bedeutung hat.

Beweis durch vollständige Induktion (80). Denn

o. der Satz ist wahr für $n=1$. In diesem Falle besteht nämlich nach 102 das System $Z_{n}$ aus der einzigen Zahl 1, und die Abbildung $\psi_{1}$ ist daher schon durch II vollständig und so definiert, daß I erfüllt ist, während III gänzlich wegfällt.

б. Ist der Satz wahr für eine Zahl $n$, so zeigen wir, daß er auch für die folgende Zahl $p=n^{\prime}$ gilt, und zwar beginnen wir mit dem Nachweise, daß es nur eine einzige entsprechende Abbildung $\psi_{p}$ des Systems $Z_{p}$ geben kann. In der Tat, genügt eine Abbildung $\psi_{p}$ den Bedingungen

$$
\begin{aligned}
& \text { I'. } \psi_{p}\left(Z_{p}\right) 3 \Omega, \\
& \text { II'. } \psi_{p}(1)=\boldsymbol{\omega}, \\
& \text { III'. } \psi_{p}\left(m^{\prime}\right)=\theta \psi_{p}(m) \text {, wenn } m<p \text {, so ist in ihr nach } 21 \text {, }
\end{aligned}
$$
weil $Z_{n} 3 Z_{p}$ ist (107), auch eine Abbildung von $Z_{n}$ enthalten, welche offenbar denselben Bedingungen I, II, III genügt wie $\psi_{n}$ und folglich mit $\psi_{n}$ gänzlich übereinstimmt; für alle in $\boldsymbol{Z}_{n}$ enthaltenen, also (98) für alle Zahlen $m$, die $<p$, d. h. $\leqq n$ sind, muß daher

$$
\psi_{p}(m)=\psi_{n}(m)
$$

*) Der Deutlichkeit wegen habe ich hier und im folgenden Satze 126 die Bedingung I besonders angeführt, obwohl sie eigentlich schon eine Folge von II und III ist. 
sein, woraus als besonderer Fall auch

$$
\psi_{p}(n)=\psi_{n}(n)
$$

folgt; da ferner $p$ nach 105, 108 die einzige nicht in $Z_{n}$ enthaltene Zahl des Systems $Z_{p}$ ist, und da nach III' $^{\prime}$ und $(n)$ auch

$$
\psi_{p}(p)=\theta \psi_{n}(n)
$$

sein $\mathrm{muß}$, so ergibt sich die Richtigkeit unserer obigen Behauptung, daß es nur eine einzige, den Bedingungen $\mathrm{I}^{\prime}, \mathrm{II}^{\prime}, \mathrm{III}^{\prime}$ genügende $\mathrm{Ab}$ bildung $\psi_{p}$ des Systems $Z_{p}$ geben kann, weil $\psi_{p}$ durch die eben abgeleiteten Bedingungen $(m)$ und $(p)$ vollständig auf $\psi_{n}$ zurückgeführt ist. Wir haben nun zu zeigen, daß umgekehrt diese durch $(m)$ und $(p)$ vollständig bestimmte Abbildung $\psi_{p}$ des Systems $Z_{p}$ wirklich den Bedingungen I', II', III' genügt. Offenbar ergibt sich $\mathrm{I}^{\prime}$ aus $(m)$ und (p) mit Rücksicht auf I und darauf, $\operatorname{da} \beta \theta(\Omega) 3 \Omega$ ist. Ebenso folgt II' aus $(m)$ und II, weil die Zahl 1 nach 99 in $Z_{n}$ enthalten ist. Die Richtigkeit von III' folgt zunächst für diejenigen Zahlen $m$, welche $<n$ sind, aus $(m)$ und III, und für die einzige noch übrige Zahl $m=n$ ergibt sie sich aus $(p)$ und $(n)$. Hiermit ist vollständig dargetan, daß aus der Gültigkeit unseres Satzes für die Zahl $n$ immer auch seine Gültigkeit für die folgende Zahl $p$ folgt, w. z. b. w.

126. Satz der Definition durch Induktion. Ist eine beliebige (ähnliche oder unähnliche) Abbildung $\theta$ eines Systems $\Omega$ in sich selbst und außerdem ein bestimmtes Element $\omega$ in $\boldsymbol{\Omega}$ gegeben, so gibt es eine und nur eine Abbildung $\psi$ der Zahlenreihe $N$, welche den Bedingungen

$$
\begin{aligned}
& \text { I. } \psi(N) 3 \Omega \\
& \text { II. } \psi(1)=\omega \\
& \text { III. } \psi\left(n^{\prime}\right)=\theta \psi(n) \text { genügt, wo } n \text { jede Zahl bedeutet. }
\end{aligned}
$$

Beweis. Da, wenn es wirklich eine solche Abbildung $\psi$ gibt, in ihr nach 21 auch eine Abbildung $\psi_{n}$ des Systems $Z_{n}$ enthalten ist, welche den in 125 angegebenen Bedingungen I, II, III genügt, so muß, weil es stets eine und nur eine solche Abbildung $\psi_{n}$ gibt, notwendig

$$
\psi(n)=\psi_{n}(n)
$$

sein. Da hierdurch $\psi$ vollständig bestimmt ist, so folgt, daß es auch nur eine einzige solche Abbildung $\psi$ geben kann (vgl. den Schluß von 130). Daß umgekehrt die durch $(n)$ bestimmte Abbildung $\psi$ auch unseren Bedingungen I, II, III genügt, folgt mit Leichtigkeit aus $(n)$ 
unter Berücksichtigung der in 125 bewiesenen Eigenschaften I, II und $(p)$, w. z. b. w.

127. Satz. Unter den im vorhergehenden Satze gemachten Voraussetzungen ist

$$
\psi\left(T^{\prime}\right)=\theta \psi(T)
$$

wo $T$ irgendeinen Teil der Zahlenreihe $N$ bedeutet.

Beweis. Denn wenn $t$ jede Zahl des Systems $T$ bedeutet, so besteht $\psi\left(T^{\prime}\right)$ aus allen Elementen $\psi\left(t^{\prime}\right)$, und $\theta \psi(T)$ aus allen Elementen $\theta \psi(t)$; hieraus folgt unser Satz, weil (nach III in 126) $\psi\left(t^{\prime}\right)=\theta \psi(t)$ ist.

128. Satz. Behält man dieselben Voraussetzungen bei und bezeichnet man mit $\theta_{0}$ die Ketten (44), welche der Abbildung $\theta$ des Systems $\Omega$ in sich selbst entsprechen, so ist

$$
\psi(N)=\theta_{0}(\omega) \text {. }
$$

Beweis. Wir zeigen zunächst durch vollständige Induktion (80), daß

$$
\psi(N) 3 \theta_{0}(\omega)
$$

d. h. daß jedes Bild $\psi(n)$ auch Element von $\theta_{0}(\omega)$ ist. In der Tat,

○. dieser Satz ist wahr für $n=1$, weil (nach 126. II) $\psi(1)=\omega$, und weil (nach 45) $\omega 3 \theta_{0}(\omega)$ ist.

б. Ist der Satz wahr für eine Zahl $n$, ist also $\psi(n) 3 \theta_{0}(\omega)$, so ist nach 55 auch $\theta(\psi(n)) 3 \theta_{0}(\omega) ;$ d. h. (nach 126. III) $\psi\left(n^{\prime}\right) 3 \theta_{0}(\omega)$, also gilt der Satz auch für die folgende Zahl $n^{\prime}$, w. z. b. w.

Um ferner $\mathrm{zu}$ beweisen, daß jedes Element $v$ der Kette $\theta_{0}(\omega)$ in $\psi(N)$ enthalten, daß also

$$
\theta_{0}(\omega) 3 \psi(N)
$$

ist, wenden wir ebenfalls die vollständige Induktion, nämlich den auf $\Omega$ und die Abbildung $\theta$ übertragenen Satz 59 an. In der Tat,

$\varrho$. das Element $\omega$ ist $=\psi(1)$, also in $\psi(N)$ enthalten.

б. Ist $v$ ein gemeinsames Element der Kette $\theta_{0}(\omega)$ und des Systems $\psi(N)$, so ist $v=\psi(n)$, wo $n$ eine Zahl bedeutet, und hieraus folgt (nach 126. III) $\theta(v)=\theta \psi(n)=\psi\left(n^{\prime}\right)$, mithin ist auch $\theta(v)$ in $\psi(N)$ enthalten, w. z. b. w.

Aus den bewiesenen Sätzen $\psi(N) 3 \theta_{0}(\omega)$ und $\theta_{0}(\omega) 3 \psi(N)$ folgt (nach 5) $\psi(N)=\theta_{0}(\omega)$, w. z. b. w.

129. Satz. Unter denselben Voraussetzungen ist allgemein

$$
\psi\left(n_{0}\right)=\theta_{0}(\psi(n)) .
$$


Beweis durch vollständige Induktion 80. Denn

@. der Satz gilt zufolge 128 für $n=1$, weil $1_{0}=N$ und $\psi(1)=\omega$ ist.

б. Ist der Satz wahr für eine Zahl $n$, so folgt

$$
\theta\left(\psi\left(n_{0}\right)\right)=\theta\left(\theta_{0}(\psi(n))\right)
$$

da nun nach 127,75

und nach 57, 126. III

$$
\theta\left(\psi\left(n_{0}\right)\right)=\psi\left(n_{0}^{\prime}\right)
$$

$$
\theta\left(\theta_{0}(\psi(n))\right)=\theta_{0}(\theta(\psi(n)))=\theta_{0}\left(\psi\left(n^{\prime}\right)\right)
$$

ist, so ergibt sich

$$
\psi\left(n_{0}^{\prime}\right)=\theta_{0}\left(\psi\left(n^{\prime}\right)\right)
$$

d. h. der Satz gilt auch für die auf $n$ folgende Zahl $n^{\prime}$, w. z. b. w.

130. Bemerkung. Bevor wir zu den wichtigsten Anwendungen des in 126 bewiesenen Satzes der Definition durch Induktion übergehen ( $\S 10$ bis 14), verlohnt es sich der Mühe, auf einen Umstand aufmerksam zu machen, durch welchen sich derselbe von dem in 80 , oder vielmehr schon in 59, 60 bewiesenen Satze der Demonstration durch Induktion wesentlich unterscheidet, so nahe auch die Verwandtschaft zwischen jenem und diesem zu sein scheint. Während nämlich der Satz 59 ganz allgemein für jede Kette $A_{0}$ gilt, wo $A$ irgendein Teil eines durch eine beliebige Abbildung $\varphi$ in sich selbst abgebildeten Systems $S$ ist ( $(4)$, so verhält es sich ganz anders mit dem Satze 126, welcher nur die Existenz einer widerspruchsfreien (oder eindeutigen) Abbildung $\psi$ des einfach unendlichen Systems $1_{0}$ behauptet. Wollte man in dem letzteren Satze (unter Beibehaltung der Voraussetzungen über $\Omega$ und $\theta$ ) an Stelle der Zahlenreihe $1_{0}$ eine beliebige Kette $A_{0}$ aus einem solchen System $S$ setzen, und etwa eine Abbildung $\psi$ von $A_{0}$ in $\Omega$ auf ähnliche Weise wie in 126. II, III dadurch definieren, daß

@. jedem Element $a$ von $A$ ein bestimmtes aus $\Omega$ gewähltes Element $\psi(\alpha)$ entsprechen, und

б. daß für jedes in $A_{0}$ enthaltene Element $n$ und dessen Bild $n^{\prime}=\varphi(n)$ die Bedingung $\psi\left(n^{\prime}\right)=\theta \psi(n)$ gelten soll,

so würde sehr häufig der Fall eintreten, daß es eine solche Abbildung $\psi$ gar nicht gibt, weil diese Bedingungen $\varrho, \sigma$ selbst dann noch in Widerspruch miteinander geraten können, wenn man auch die in @ enthaltene Wahlfreiheit von vornherein der Bedingung $\sigma$ gemäß beschränkt. Ein Beispiel wird genügen, um sich hiervon zu überzeugen. 
Ist das aus den verschiedenen Elementen $a$ und $b$ bestehende System $S$ durch $\varphi$ so in sich selbst abgebildet, daß $a^{\prime}=b, b^{\prime}=a$ wird, so ist offenbar $a_{0}=b_{0}=S$; es sei ferner das aus den verschiedener Elementen $\alpha, \beta$ und $\gamma$ bestehende System $\Omega$ durch $\theta$ so in sich selbst abgebildet, daß $\theta(\alpha)=\beta, \theta(\beta)=\gamma, \theta(\gamma)=\alpha$ wird; verlangt man nun eine solche Abbildung $\psi$ von $a_{0}$ in $\Omega$, daß $\psi(a)=\alpha$ und außerdem für jedes in $a_{0}$ enthaltene Element $n$ immer $\psi\left(n^{\prime}\right)=\theta \psi(n)$ wird, so stößt man auf einen Widerspruch; denn für $n=a$ ergibt $\operatorname{sich} \psi(b)=\theta(\alpha)=\beta$, und hieraus folgt für $n=b$, daß $\psi(a)=\theta(\beta)=\gamma$ sein müßte, während doch $\psi(a)=\alpha$ war.

Gibt es aber eine Abbildung $\psi$ von $A_{0}$ in $\Omega$, welche den obigen Bedingungen $\varrho$, $\sigma$ ohne Widerspruch genügt, so folgt aus 60 leicht, daß sie vollständig bestimmt ist; denn wenn die Abbildung $\chi$ denselben Bedingungen genügt, so ist allgemein $\chi(n)=\psi(n)$, weil dieser Satz zufolge $\varrho$ für alle in $A$ enthaltenen Elemente $n=a$ gilt, und weil er, wenn er für ein Element $n$ von $A_{0}$ gilt, zufolge $\sigma$ anch für dessen Bild $n^{\prime}$ gelten muß.

131. Um die Tragweite unseres Satzes 126 ins Licht zu setzen, wollen wir hier eine Betrachtung einfügen, die auch für andere Untersuchungen, z. B. für die sogenannte Gruppentheorie, nützlich ist.

Wir betrachten ein System $\boldsymbol{\Omega}$, dessen Elemente eine gewisse Verbindung gestatten in der Art, daß aus einem Elemente $\boldsymbol{v}$ durch Einwirkung eines Elementes $\omega$ immer wieder ein bestimmtes Element desselben Systems $\boldsymbol{\Omega}$ entspringt, welches mit $\boldsymbol{\omega} \cdot \boldsymbol{v}$ oder $\boldsymbol{\omega} \boldsymbol{\nu}$ bezeichnet werden mag und im allgemeinen von $v \omega \mathrm{zu}$ unterscheiden ist. Man kann dies auch so auffassen, daß jedem bestimmten Elemente $\omega$ eine bestimmte, etwa durch $\dot{\omega} \mathrm{zu}$ bezeichnende Abbildung des Systems $\boldsymbol{\Omega}$ in sich selbst entspricht, insofern jedes Element $\nu$ das bestimmte Bild $\dot{\omega}(\nu)=\omega v$ liefert. Wendet man auf dieses System $\Omega$ und dessen Element $\omega$ den Satz 126 an, indem man zugleich die dort mit $\theta$ bezeichnete Abbildung durch $\dot{\omega}$ ersetzt, so entspricht jeder Zahl $n$ ein bestimmtes, in $\Omega$ enthaltenes Element $\psi(n)$, das jetzt durch das Symbol $\boldsymbol{\omega}^{n}$ bezeichnet werden mag und bisweilen die $n$-te Potenz von $\omega$ genannt wird; dieser Begriff ist vollständig erklärt durch die ihm auferlegten Bedingungen

$$
\begin{gathered}
\text { II. } \boldsymbol{\omega}^{1}=\omega, \\
\text { III. } \boldsymbol{\omega}^{n^{\prime}}=\omega \boldsymbol{\omega}^{n},
\end{gathered}
$$

und seine Existenz ist durch den Beweis des Satzes 126 gesichert. 
Ist die obige Verbindung der Elemente außerdem so beschaffen, daß für beliebige Elemente $\mu, v, \omega$ stets $\omega(\nu \mu)=(\omega v) \mu$ ist, so gelten auch die Sätze

$$
\omega^{n^{\prime}}=\omega^{n} \omega, \quad \omega^{m} \omega^{n}=\omega^{n} \omega^{m},
$$

deren Beweise leicht durch vollständige Induktion (80) zu führen sind und dem Leser überlassen bleiben mögen.

Die vorstehende allgemeine Betrachtung läßt sich unmittelbar auf folgendes Beispiel anwenden. Ist $S$ ein System von beliebigen Elementen, und $\Omega$ das zugehörige System, dessen Elemente die sämtlichen Abbildungen $v$ von $S$ in sich selbst sind (36), so lassen diese Elemente sich nach 25 immer zusammensetzen, weil $v(S) 3 S$ ist, und die aus solchen Abbildungen $\nu$ und $\omega$ zusammengesetzte Abbildung $\omega \nu$ ist selbst wieder Element von $\Omega$. Dann sind auch alle Elemente $\boldsymbol{\omega}^{n}$ Abbildungen von $S$ in sich selbst, und man sagt, sie entstehen durch Wiederholung der Abbildung $\omega$. Wir wollen nun einen einfachen Zusammenhang hervorheben, der zwischen diesem Begriffe und dem in 44 erklärten Begriffe der Kette $\omega_{0}(A)$ besteht, wo $A$ wieder irgendeinen Teil von $S$ bedeutet. Bezeichnet man der Kürze halber das durch die Abbildung $\omega^{n}$ erzeugte Bild $\omega^{n}(A)$ mit $A_{n}$, so folgt aus III, 25, daß $\omega\left(A_{n}\right)=A_{n^{\prime}}$ ist. Hieraus ergibt sich leicht durch vollständige Induktion (80), daß alle diese Systeme $A_{n}$ Teile der Kette $\omega_{0}(A)$ sind; denn

@. diese Behauptung gilt zufolge 50 für $n=1$, und

б. wenn sie für eine Zahl $n$ gilt, so folgt aus 5. und aus $A_{n^{\prime}}=\omega\left(A_{n}\right)$, daß sie auch für die folgende $n^{\prime}$ gilt, w. z. b. w. Da ferner nach 45 auch $A 3 \omega_{0}(A)$ ist, so ergibt sich aus 10 , daß auch das aus $A$ und aus allen Bildern $A_{n}$ zusammengesetzte System $K$ Teil von $\omega_{0}(A)$ ist. Umgekehrt, da (nach 23) $\omega(K)$ aus $\omega(A)=A_{1}$ und aus allen Systemen $\omega\left(A_{n}\right)=A_{n^{\prime}}$, also (nach 78 ) aus allen Systemen $A_{n}$ zusammengesetzt ist, welche nach 9 Teile von $K$ sind, so ist (nach 10) $\omega(K) 3 K$, d. h. $K$ ist eine Kette (37), und da (nach 9) $A 3 K$ ist, so folgt nach 47 , daß auch $\omega_{0}(A) 3 K$ ist. Mithin ist $\omega_{0}(A)=K$, d. h. es besteht folgender Satz: Ist $\omega$ eine Abbildung eines Systems $S$ in sich selbst, und $A$ irgendein Teil von $S$, so ist die der Abbildung $\omega$ entsprechende Kette von $A$ zusammengesetzt aus $A$ und allen durch Wiederholung von $\omega$ entstehenden Bildern $\omega^{n}(A)$. Wir empfehlen dem Leser, mit dieser Auffassung einer Kette zu den früheren Sätzen 57, 58 zurückzukenren. 
$\S 10$.

Die Klasse der einfach unendlichen Systeme.

132. Satz. Alle einfach unendlichen Systeme sind der Zahlenreihe $N$ und folglich (nach 33 ) auch einander ähnlich.

Beweis. Es sei das einfach unendliche System $\boldsymbol{\Omega}$ durch die Abbildung $\theta$ geordnet (71), und es sei $\omega$ das hierbei auftretende Grundelement von $\Omega$; bezeichnen wir mit $\theta_{0}$ wieder die der Abbildung $\theta$ entsprechenden Ketten (44), so gilt nach 71 folgendes:

$\alpha . \quad \theta(\Omega) 3 \Omega$.

ק. $\Omega=\theta_{0}(\omega)$.

$\gamma$. $\omega$ ist nicht in $\theta(\Omega)$ enthalten.

$\delta$. Die Abbildung $\theta$ ist eine ähnliche.

Bedeutet nun $\psi$ die in 126 definierte Abbildung der Zahlenreihe $N$, so folgt aus $\beta$ und 128 zunächst

$$
\psi(N)=\Omega,
$$

und wir haben daher nach 32 nur noch zu zeigen, daß $\psi$ eine ähnliche Abbildung ist, d. h. (26) daß verschiedenen Zahlen $m, n$ auch verschiedene Bilder $\psi(m), \psi(n)$ entsprechen. Der Symmetrie wegen dürfen wir nach 90 annehmen, es sei $m>n$, also $m 3 n_{0}^{\prime}$, und der zu beweisende Satz kommt darauf hinaus, daß $\psi(n)$ nicht in $\psi\left(n_{0}^{\prime}\right)$, also (nach 127) nicht in $\theta \psi\left(n_{0}\right)$ enthalten ist. Dies beweisen wir für jede Zahl $n$ durch vollständige Induktion (80). In der Tat,

@. dieser Satz gilt nach $\gamma$ für $n=1$, weil $\psi(1)=\omega$ und $\psi\left(1_{0}\right)$ $=\psi(N)=\Omega$ ist.

б. Ist der Satz wahr für eine Zahl $n$, so gilt er auch für die folgende Zahl $n^{\prime}$; denn wäre $\psi\left(n^{\prime}\right)$, d. h. $\theta \psi(n)$ in $\theta \psi\left(n_{0}^{\prime}\right)$ enthalten, so müßte (nach $\delta$ und 27) auch $\psi(n)$ in $\psi\left(n_{0}^{\prime}\right)$ enthalten sein, während unsere Annahme gerade das Gegenteil besagt, w. z. b.w.

133. Satz. Jedes System, welches einem einfach unendliclien System und folglich (nach 132, 33) auch der Zahlenreihe $N$ ähnlich ist, ist einfach unendlich.

Beweis. Ist $\Omega$ ein der Zahlenreihe $N$ ähnliches System, so gibt es nach 32 eine solche ähnliche Abbildung $\psi$ von $N$, daß

wird; dann setzen wir

$$
\text { I. } \psi(N)=\Omega
$$

II. $\psi(1)=\omega$. 
Bezeichnet man nach 26 mit $\bar{\psi}$ die umgekehrte, ebenfalls ähnliche Abbildung von $\Omega$, so entspricht jedem Elemente $\boldsymbol{v}$ von $\Omega$ eine bestimmte Zahl $\bar{\psi}(v)=n$, nämlich diejenige, deren Bild $\psi(n)=v$ ist. Da nun dieser Zahl $n$ eine bestimmte folgende Zahl $\varphi(n)=n^{\prime}$, und dieser wieder ein bestimmtes Element $\psi\left(n^{\prime}\right)$ in $\Omega$ entspricht, so gehört zu jedem Elemente $v$ des Systems $\Omega$ auch ein bestimmtes Element $\psi\left(n^{\prime}\right)$ desselben Systems, das wir als Bild von $v$ mit $\theta(v)$ bezeichnen wollen. Hierdurch ist eine Abbildung $\theta$ von $\Omega$ in sich selbst vollständig bestimmt*), und um unseren Satz zu beweisen, wollen wir zeigen, daß $\Omega$ durch $\theta$ als einfach unendliches System geordnet ist (71), d. h. daß die in dem Beweise von 132 angegebenen Bedingungen $\alpha, \beta, \gamma, \delta$ sämtlich erfüllt sind. Zunächst leuchtet $\alpha$ aus der Definition von $\theta$ unmittelbar ein. Da ferner jeder Zahl $n$ ein Element $v=\psi(n)$ entspricht, für welches $\theta(v)=\psi\left(n^{\prime}\right)$ wird, so ist allgemein

$$
\text { III. } \psi\left(n^{\prime}\right)=\theta \psi(n) \text {, }
$$

und hieraus in Verbindung mit I, II, $\alpha$ ergibt sich, daß die Abbildungen $\theta, \psi$ alle Bedingungen des Satzes 126 erfüllen; mithin folgt $\beta$ aus 128 und I. Nach 127 und I ist ferner

$$
\psi\left(N^{\prime}\right)=\theta \psi(N)=\theta(\Omega),
$$

und hieraus in Verbindung mit II und der Ähnlichkeit der Abbildung $\psi$ folgt $\gamma$, weil sonst $\psi(1)$ in $\psi\left(N^{\prime}\right)$, also (nach 27) die Zahl 1 in $N^{\prime}$ enthalten sein müßte, was (nach 71. $\gamma$ ) nicht der Fall ist. Wenn endlich $\mu, \nu$ Elemente von $\Omega$, und $m, n$ die entsprechenden Zahlen bedeuten, deren Bilder $\psi(m)=\mu, \psi(n)=\boldsymbol{\nu}$ sind, so folgt aus der Annahme $\theta(\mu)=\theta(\nu)$ nach dem Obigen, daß $\psi\left(m^{\prime}\right)=\psi\left(n^{\prime}\right)$, hieraus wegen der Ähnlichkeit von $\psi, \varphi$, daß $m^{\prime}=n^{\prime}, m=n$, also auch $\mu=\nu$ ist; mithin gilt auch $\delta$, w. z. b.w.

134. Bemerkung. Zufolge der beiden vorhergehenden Sätze 132, 133 bilden alle einfach unendlichen Systeme eine Klasse im Sinne von 34. Zugleich leuchtet mit Rücksicht auf 71,73 ein, daß jeder Satz über die Zahlen, d. h. über die Elemente $n$ des durch die Abbildung $\varphi$ geordneten einfach unendlichen Systems $N$, und zwar jeder solche Satz, in welchem von der besonderen Beschaffenheit der Elemente $n$ gänzlich abgesehen wird und nur von solchen $\mathrm{Be}$ griffen die Rede ist, die aus der Anordnung $\varphi$ entspringen, ganz

*) Offenbar ist $\theta$ die nach 25 aus $\bar{\psi}, \varphi, \psi$ zusammengesetzte Abbildung $\psi \varphi \bar{\psi}$. 
allgemeine Gültigkeit auch für jedes andere durch eine Abbildung $\theta$ geordnete einfach unendliche System $\boldsymbol{\Omega}$ und dessen Elemente $\boldsymbol{v}$ besitzt, und daß die Übertragung von $N$ auf $\Omega$ (z. B. auch die Übersetzung eines arithmetischen Satzes aus einer Sprache in eine andere) durch die in 132,133 betrachtete Abbildung $\psi$ geschieht, welche jedes Element $n$ von $N$ in ein Element $\nu$ von $\Omega$, nämlich in $\psi(n)$ verwandelt. Dieses Element $v$ kann man das $n$-te Element von $\Omega$ nennen, und hiernach ist die Zahl $n$ selbst die $n$-te Zahl der Zahlenreihe $N$. Dieselbe Bedeutung, welche die Abbildung $\varphi$ für die Gesetze im Gebiete $N$ besitzt, insofern jedem Elemente $n$ ein bestimmtes Element $\varphi(n)=n^{\prime}$ folgt, kommt nach der durch $\psi$ bewirkten Verwandlung der Abbildung $\theta$ zu für dieselben Gesetze im Gebiete $\Omega$, insofern dem durch Verwandlung von $n$ entstandenen Elemente $\boldsymbol{\nu}=\psi(n)$ das durch Verwandlung von $n^{\prime}$ entstandene Element $\theta(\nu)=\psi\left(n^{\prime}\right)$ folgt; man kann daher mit Recht sagen, daß $\varphi$ durch $\psi$ in $\theta$ verwandelt wird, was sich symbolisch durch $\theta=\psi \varphi \bar{\psi}, \varphi=\bar{\psi} \theta \psi$ ausdrückt. Durch diese Bemerkungen wird, wie ich glaube, die in 73 aufgestellte Erklärung des Begriffes der Zahlen vollständig gerechtfertigt. Wir gehen nun zu ferneren Anwendungen des Satzes 126 über.

\section{$\S 11$.}

Addition der Zahlen.

135. Erklärung. Es liegt nahe, die im Satze 126 dargestellte Definition einer Abbildung $\psi$ der Zahlenreihe $N$ oder der durch dieselbe bestimmten Funktion $\psi(n)$ auf den Fall anzuwenden, wo das dort mit $\Omega$ bezeichnete System, in welchem das Bild $\psi(N)$ enthalten sein soll, die Zahlenreihe $N$ selbst ist, weil für dieses System $\Omega$ schon eine Abbildung $\theta$ von $\Omega$ in sich selbst vorliegt, nämlich diejenige Abbildung $\varphi$, durch welche $N$ als einfach unendliches System geordnet ist $(71,73)$. Dann wird also $\Omega=N, \theta(n)=\varphi(n)=n^{\prime}$, mithin

$$
\text { I. } \psi(N) 3 N \text {, }
$$

und es bleibt, um $\psi$ vollständig zu bestimmen, nur noch übrig, das Element $\boldsymbol{\omega}$ aus $\boldsymbol{\Omega}, \mathrm{d}$. h. aus $N$ nach Belieben zu wählen. Nehmen wir $\omega=1$, so wird $\psi$ offenbar die identische Abbildung (21) von $N$, weil den Bedingungen

$$
\psi(1)=1, \quad \psi\left(n^{\prime}\right)=(\psi(n))^{\prime}
$$


allgemein durch $\psi(n)=n$ genügt wird. Soll also eine andere Abbildung $\psi$ von $N$ erzeugt werden, so muß für $\omega$ eine von 1 verschiedene, nach 78 in $N^{\prime}$ enthaltene Zahl $m^{\prime}$ gewählt werden, wo $m$ selbst irgendeine Zahl bedeutet; da die Abbildung $\psi$ offenbar von der Wahl dieser Zahl $m$ abhängig ist, so bezeichnen wir das entsprechende Bild $\psi(n)$ einer beliebigen Zahl $n$ durch das Symbol $m+n$ und nennen diese Zahl die Summe, welche aus der Zahl $m$ durch Addition der Zahl $n$ entsteht, oder kurz die Summe der Zahlen $m, n$. Dieselbe ist daher nach 126 vollständig bestimmt durch die Bedingungen*)

$$
\begin{aligned}
\text { II. } & m+1=m^{\prime}, \\
\text { III. } & m+n^{\prime}=(m+n)^{\prime} .
\end{aligned}
$$

136. Satz. Es ist $m^{\prime}+n=m+n^{\prime}$.

Beweis durch vollständige Induktion (80). Denn

@. der Satz ist wahr für $n=1$, weil (nach 135. II)

$$
m^{\prime}+1=\left(m^{\prime}\right)^{\prime}=(m+1)^{\prime}
$$

und (nach 135. III) $(m+1)^{\prime}=m+1^{\prime}$ ist.

б. Ist der Satz wahr für eine Zahl $n$, und setzt man die folgende Zahl $n^{\prime}=p$, so ist $m^{\prime}+n=m+p$, also auch $\left(m^{\prime}+n\right)^{\prime}=(m+p)^{\prime}$, woraus (nach 135. III) $m^{\prime}+p=m+p^{\prime}$ folgt; mithin gilt der Satz auch für die folgende Zahl $p$, w. z. b.w.

137. Satz. Es ist $m^{\prime}+n=(m+n)^{\prime}$.

Der Beweis folgt aus 136 und 135. III.

138. Satz. Es ist $1+n=n^{\prime}$.

Beweis durch vollständige Induktion (80). Denn

o. der Satz ist nach 135. II wahr für $n=1$.

б. Gilt der Satz für eine Zahl $n$, und setzt man $n^{\prime}=p$, so ist $1+n=p$, also auch $(1+n)^{\prime}=p^{\prime}$, mithin (nach 135. III) $1+p=p^{\prime}$, d. h. der Satz gilt auch für die folgende Zahl $p$, w. z. b. w. 139. Satz. Es ist $1+n=n+1$.

*) Die obige, unmittelbar auf den Satz 126 gegründete Erklärung der Addition scheint mir die einfachste zu sein. Mit Zuziehung des in 131 entwickelten Begriffes kann man aber die Summe $m+n$ auch durch $\varphi^{n}(m)$ oder auch durch $\varphi^{m}(n)$ definieren, wo $\varphi$ wieder die obige Bedeutung hat. Um die vollständige Übereinstimmung dieser Definitionen mit der obigen zu beweisen, braucht man nach 126 nur zu zeigen, daß, wenn $\varphi^{n}(m)$ oder $\varphi^{m}(n)$ mit $\psi(n)$ bezeichnet wird, die Bedingungen $\psi(1)=m^{\prime}, \psi\left(n^{\prime}\right)=\varphi \psi(n)$ erfüllt sind, was mit Hilfe der vollständigen Induktion (80) unter Zuziehung von 131 leicht gelingt. 
Der Beweis folgt aus 138 und 135. II.

140. Satz. Es ist $m+n=n+m$.

Beweis durch vollständige Induktion (80). Denn

@. der Satz ist nach 139 wahr für $n=1$.

б. Gilt der Satz für eine Zahl $n$, so folgt daraus auch $(m+n)^{\prime}$ $=(n+m)^{\prime}$, d. h. (nach 135. III) $m+n^{\prime}=n+m^{\prime}$, mithin (nach 136) $m+n^{\prime}=n^{\prime}+m$; mithin gilt der Satz auch für die folgende Zahl $n^{\prime}$, w. z. b. w.

141. Satz. Es ist $(l+m)+n=l+(m+n)$.

Beweis durch vollständige Induktion (80). Denn

@. der Satz ist wahr für $n=1$, weil (nach 135 . II, III, II) $(l+m)+1=(l+m)^{\prime}=l+m^{\prime}=l+(m+1)$ ist.

б. Gilt der Satz für eine Zahl $n$, so folgt daraus auch $((l+m)+n)^{\prime}=(l+(m+n))^{\prime}$, d. h. $($ nach 135. III)

$$
(l+m)+n^{\prime}=l+(m+n)^{\prime}=l+\left(m+n^{\prime}\right),
$$

also gilt der Satz auch für die folgende Zahl $n^{\prime}$, w. z. b. w.

142. Satz. Es ist $m+n>m$.

Beweis durch vollständige Induktion (80). Denn

@. der Satz ist nach 135 . II und 91 wahr für $n=1$.

б. Gilt der Satz für eine Zahl $n$, so gilt er nach 95 auch für die folgende Zahl $n^{\prime}$, weil (nach 135. III und 91)

ist, w. z. b.w.

$$
m+n^{\prime}=(m+n)^{\prime}>m+n
$$

143. Satz. Die Bedingungen $m>a$ und $m+n>a+n$ sind gleichwertig.

Beweis durch vollständige Induktion (80). Denn

@. der Satz gilt zufolge 135. II und 94 für $n=1$.

б. Gilt der Satz für eine Zahl $n$, so gilt er auch für die folgende Zahl $n^{\prime}$, weil die Bedingung $m+n>a+n$ nach 94 mit $(m+n)^{\prime}>(a+n)^{\prime}$, also nach 135 . III auch mit

$$
m+n^{\prime}>a+n^{\prime}
$$

gleichwertig ist, w. z. b.w.

144. Satz. Ist $m>a$ und $n>b$, so ist auch

$$
m+n>a+b \text {. }
$$

Beweis. Denn aus unseren Voraussetzungen folgt (nach 143) $m+n>a+n$ und $n+a>b+a$ oder, was nach 140 dasselbe ist, $a+n>a+b$, woraus sich der Satz nach 95 ergibt.

145. Satz. Ist $m+n=a+n$, so ist $m=a$. 
Beweis. Denn wenn $m$ nicht $=a$, also nach 90 entweder $m>a$ oder $m<a$ ist, so ist nach 143 entsprechend $m+n>a+n$ oder $m+n<a+n$, also kann (nach 90) $m+n$ gewiß nicht $=a+n$ sein, w. z. b.w.

146. Satz. Ist $l>n$, so gibt es eine und (nach 145) nur eine Zahl $m$, welche der Bedingung $m+n=l$ genügt.

Beweis durch vollständige Induktion (80). Denn

@. der Satz ist wahr für $n=1$. In der Tat, wenn $l>1$, d.h. (89) wenn $l$ in $N^{\prime}$ enthalten, also das Bild $m^{\prime}$ einer Zahl $m$ ist, so folgt aus 135 . II, daß $l=m+1$ ist, w. z. b.w.

б. Gilt der Satz für eine Zahl $n$, so zeigen wir, daß er auch für die folgende Zahl $n^{\prime}$ gilt. In der Tat, wenn $l>n^{\prime}$ ist, so ist nach 91, 95 auch $l>n$, und folglich gibt es eine Zahl $k$, welche der Bedingung $l=k+n$ genïgt; da dieselbe nach 138 verschieden von 1 ist (weil sonst $l=n^{\prime}$ wäre), so ist sie nach 78 das Bild $m^{\prime}$ einer Zahl $m$, und folglich ist $l=m^{\prime}+n$, also nach 136 auch $l=m+n^{\prime}$, w.z. b.w.

\section{$\S 12$.}

Multiplikation der Zahlen.

147. Erklärung. Nachdem im vorhergehenden $\S 11$ ein unendliches System neuer Abbildungen der Zahlenreihe $N$ in sich selbst gefunden ist, kann man jede derselben nach 126 wieder benutzen, um abermals neue Abbildungen $\psi$ von $N$ zu erzeugen. Indem man daselbst $\dot{\Omega}=N$ und $\theta(n)=m+n=n+m$ setzt, wo $m$ eine bestimmte Zahl, wird jedenfalls wieder

$$
\text { I. } \psi(N) 3 N \text {, }
$$

und es bleibt, um $\psi$ vollständig zu bestimmen, nur noch übrig, das Element $\omega$ aus $N$ nach Belieben zu wählen. Der einfachste Fali tritt dann ein, wenn man diese $\mathrm{Wahl}$ in eine gewisse Übereinstimmung mit der Wahl von $\theta$ bringt, indem man $\omega=m$ setzt. Da die hierdurch vollständig bestimmte Abbildung $\psi$ von dieser Zahl $m$ abhängt, so bezeichnen wir das entsprechende Bild $\psi(n)$ einer beliebigen Zahl $n$ durch das Symbol $m \times n$ oder $m . n$ oder $m n$, und nennen diese Zahl das Produkt, welches aus der Zahl $m$ durch Multiplikation mit der Zahl $n$ entsteht, oder kurz das Produkt dor 
Zahlen $m, n$. Dasselbe ist daher nach 126 vollständig bestimmt durch die Bedingungen

$$
\begin{aligned}
\text { II. } & m \cdot 1=m, \\
\text { III. } & m n^{\prime}=m n+m .
\end{aligned}
$$

148. Satz. Es ist $m^{\prime} n=m n+n$.

Beweis durch vollständige Induktion (80). Denn

@. der Satz ist nach 147. II und 135. II wahr für $n=1$.

б. Gilt der Satz für eine Zahl $n$, so folgt

$$
m^{\prime} n+m^{\prime}=(m n+n)+m^{\prime}
$$

und hieraus (nach 147. III, 141, 140, 136, 141, 147. III)

$$
\begin{aligned}
& m^{\prime} n^{\prime}=m n+\left(n+m^{\prime}\right)=m n+\left(m^{\prime}+n\right) \\
= & m n+\left(m+n^{\prime}\right)=(m n+m)+n^{\prime}=m n^{\prime}+n^{\prime} ;
\end{aligned}
$$

also gilt der Satz auch für die folgende Zahl $n^{\prime}$, w. z. b. w.

149. Satz. Es ist $1 . n=n$.

Beweis durch vollständige Induktion (80). Denn

@. der Satz ist nach 147. II wahr für $n=1$.

б. Gilt der Satz für eine Zahl $n$, so folgt $1 . n+1=n+1$, d. h. (nach 147. III, 135. II) $1 . n^{\prime}=n^{\prime}$, also gilt der Satz auch für die folgende Zahl $n^{\prime}$, w. z. b. w.

150. Satz. Es ist $m n=n m$.

Beweis durch vollständige Induktion (80). Denn

@. der Satz gilt nach 147. II, 149 für $n=1$.

б. Gilt der Satz für eine Zahl $n$, so folgt

$$
m n+m=n m+m,
$$

d. h. (nach 147. III, 148) $m n^{\prime}=n^{\prime} m$, also gilt der Satz auch für die folgende Zahl $n^{\prime}$, w. z. b.w.

151. Satz. Es ist $l(m+n)=l m+l n$.

Beweis durch vollständige Induktion (80). Denn

@. der Satz ist nach 135. II, 147. III, 147. II wahr für $n=1$.

б. Gilt der Satz für eine Zahl $n$, so folgt

$$
l(m+n)+l=(l m+l n)+l
$$

nach 147. III, 135. III ist aber

$$
l(m+n)+l=l(m+n)^{\prime}=l\left(m+n^{\prime}\right)
$$

und nach 141,147 . III ist

$$
(l m+l n)+l=l m+(l n+l)=l m+l n^{\prime}
$$

mithin ist $l\left(m+n^{\prime}\right)=l m+l n^{\prime}$, d. h. der Satz gilt auch für die folgende Zahl $n^{\prime}$, w. z. b.w. 
152. Satz. Es ist $(m+n) l=m l+n l$.

Der Beweis folgt aus 151, 150.

153. Satz. Es ist $(l m) n=l(m n)$.

Beweis durch vollständige Induktion (80). Denn

@. der Satz gilt nach 147. II für $n=1$.

б. Gilt der Satz für eine Zahl $n$, so folgt

$$
(l m) n+l m=l(m n)+l m,
$$

d. h. (nach 147. III, 151, 147. III)

$$
(l m) n^{\prime}=l(m n+m)=l\left(m n^{\prime}\right),
$$

also gilt der Satz auch für die folgende Zahl $n^{\prime}$, w. z. b.w.

154. Bemerkung. Hätte man in 147 keine Beziehung zwischen $\omega$ und $\theta$ angenommen, sondern $\omega=k, \theta(n)=m+n$ gesetzt, so würde hieraus nach 126 eine weniger einfache Abbildung $\psi$ der Zahlenreihe $N$ entstanden sein; für die Zahl 1 würde $\psi(1)=k$, und für jede andere, also in der Form $n^{\prime}$ enthaltene Zahl würde $\psi\left(n^{\prime}\right)=m n+k$; denn hierdurch wird, wovon man sich mit $\mathrm{Zu}$ ziehung der vorhergehenden Sätze leicht überzeugt, die Bedingung $\psi\left(n^{\prime}\right)=\theta \psi(n)$, d. h. $\psi\left(n^{\prime}\right)=m+\psi(n)$ für alle Zahlen $n$ erfüllt.

\section{$\S 13$.}

Potenzierung der Zahlen.

155. Erklärung. Wenn man in dem Satze 126 wieder $\Omega=N$, ferner $\omega=a, \theta(n)=a n=n a$ setzt, so entsteht eine Abbildung $\psi$ von $N$, welche abermals der Bedingung

\section{I. $\psi(N) 3 N$}

genügt; das entsprechende Bild $\psi(n)$ einer beliebigen Zahl $n$ bezeichnen wir mit dem Symbol $a^{n}$ und nennen diese Zahl eine Potenz der Basis $a$, während $n$ der Exponent dieser Potenz von $a$ heißt. Dieser Begriff ist daher vollständig bestimmt durch die Bedingungen

$$
\begin{aligned}
& \text { II. } a^{1}=a, \\
& \text { III. } a^{n^{\prime}}=a \cdot a^{n}=a^{n} \cdot a \text {. }
\end{aligned}
$$

156. Satz. Es ist $a^{m+n}=a^{m} \cdot a^{n}$.

Beweis durch vollständige Induktion (80). Denn

@. der Satz gilt nach 135. II, 155. III, 155. II für $n=1$.

б. Gilt der Satz für eine Zahl $n$, so folgt

$$
a^{m+n} \cdot a=\left(a^{m} \cdot a^{n}\right) a ;
$$


nach 155. III, 135. III ist aber $a^{m+n} \cdot a=a^{(m+n)^{\prime}}=a^{m+n^{\prime}}$, und nach 153,155 . III ist $\left(a^{m} \cdot a^{n}\right) a=a^{m}\left(a^{n} \cdot a\right)=a^{m} \cdot a^{n^{\prime}}$; mithin ist $a^{m+n^{\prime}}=a^{m} \cdot a^{n^{\prime}}$, d. h. der Satz gilt auch für die folgende Zahl $n^{\prime}$, w. z. b.w.

157. Satz. Es ist $\left(a^{m}\right)^{n}=a^{m n}$.

Beweis durch vollständige Induktion (80). Denn

@. der Satz gilt nach 155. II, 147. II für $n=1$.

б. Gilt der Satz für eine Zahl $n$, so folgt

$$
\left(a^{m}\right)^{n} \cdot a^{m}=a^{m n} \cdot a^{m}
$$

nach 155. III ist aber $\left(a^{m}\right)^{n} \cdot a^{m}=\left(a^{m}\right)^{n^{\prime}}$, und nach 156, 147. III ist $a^{m n} \cdot a^{m}=a^{m n+m}=a^{m n^{\prime}}$; mithin ist $\left(a^{m}\right)^{n^{\prime}}=a^{m n^{\prime}}$, d. h. der Satz gilt auch für die folgende Zahl $n^{\prime}$, w. z. b. w.

158. Satz. Es ist $(a b)^{n}=a^{n} \cdot b^{n}$.

Beweis durch vollständige Induktion (80). Denn

@. der Satz gilt nach 155. II für $n=1$.

б. Gilt der Satz für eine Zahl $n$, so folgt nach 150,153 , 155. III auch $(a b)^{n} \cdot a=a\left(a^{n} \cdot b^{n}\right)=\left(a \cdot a^{n}\right) b^{n}=a^{n^{\prime}} \cdot b^{n}$, und hieraus $\left((a b)^{n} \cdot a\right) b=\left(a^{n^{\prime}} \cdot b^{n}\right) b$; nach 153,155 . III ist aber $\left((a b)^{n} \cdot a\right) b$ $=(a b)^{n} \cdot(a b)=(a b)^{n^{\prime}}$, und ebenso

$$
\left(a^{n^{\prime}} \cdot b^{n}\right) b=a^{n^{\prime}} \cdot\left(b^{n} \cdot b\right)=a^{n^{\prime}} \cdot b^{n^{\prime}}
$$

mithin ist $(a b)^{n^{\prime}}=a^{n^{\prime}} \cdot b^{n^{\prime}}$, d. h. der Satz gilt auch für die folgende Zahl $n^{\prime}$, w. z. b.w.

\section{$\S 14$.}

Anzahl der Elemente eines endlichen Systems.

159. Satz. Ist $\Sigma$ ein unendliches System, so ist jedes der in 98 erklärten Zahlensysteme $Z_{n}$ ähnlich abbildbar in $\Sigma$ (d. h. ähnlich einem Teile von $\Sigma$ ), und umgekehrt.

Beweis. Wenn $\Sigma$ unendlich ist, so gibt es nach 72 gewiß einen Teil $T$ von $\Sigma$, welcher einfach unendlich, also nach 132 der Zahlenreihe $N$ ähnlich ist, und folglich ist nach 35 jedes System $Z_{n}$ als Teil von $N$ auch einem Teile von $T$, also auch einem T'eile von $\Sigma$ ähnlich, w.z.b.w.

Der Beweis der Umkehrung - so einleuchtend dieselbe erscheinen mag - ist umständlicher. Wenn jedes System $Z_{n}$ ähnlich abbildbar in $\Sigma$ ist, so entspricht jeder Zahl $n$ eine solche ähnliche Abbildung $\alpha_{n}$ 
von $Z_{n}$, daß $\alpha_{n}\left(Z_{n}\right) 3 \Sigma$ wird. Aus der Existenz einer solchen als gegeben anzusehenden Reihe von Abbildungen $\alpha_{n}$, über die aber weiter nichts vorausgesetzt wird, leiten wir zunächst mit Hilfe des Satzes 126 die Existenz einer neuen Reihe von ebensolchen Abbildungen $\psi_{n}$ ab, welche die besondere Eigenschaft besitzt, daß jedesmal, wenn $m \leqq n$, also (nach 100) $Z_{m} 3 Z_{n}$ ist, die Abbildung $\psi_{m}$ des Teiles $Z_{m}$ in der Abbildung $\psi_{n}$ von $Z_{n}$ enthalten ist (21), d. h. daßs die Abbildungen $\psi_{m}$ und $\psi_{n}$ für alle in $Z_{m}$ enthaltenen Zahlen gänzlich miteinander übereinstimmen, also auch stets

$$
\psi_{m}(m)=\psi_{n}(m)
$$

wird. Um den genannten Satz diesem Ziele gemäß anzuwenden, verstehen wir unter $\boldsymbol{\Omega}$ dasjenige System, dessen Elemente alle überhaupt möglichen ähnlichen Abbildungen aller Systeme $Z_{n}$ in $\Sigma$ sind, und definieren mit Hilfe der gegebenen, ebenfalls in $\Omega$ enthaltenen Elemente $\alpha_{n}$ auf folgende Weise eine Abbildung $\theta$ von $\Omega$ in sich selbst. Ist $\beta$ irgendein Element von $\Omega$, also z. B. eine ähnliche Abbildung des bestimmten Systems $Z_{n}$ in $\Sigma$, so kann das System $\alpha_{n^{\prime}}\left(Z_{n^{\prime}}\right)$ nicht Teil von $\beta\left(Z_{n}\right)$ sein, weil sonst $Z_{n^{\prime}}$ nach 35 einem Teile von $Z_{n}$, also nach 107 einem echten Teile seiner selbst ähnlich, mithin unendlich wäre, was dem Satze 119 widersprechen würde; es gibt daher in $Z_{n^{\prime}}$ gewiß eine Zahl oder verschiedene Zahlen $p$ derart, daß $\alpha_{n^{\prime}}(p)$ nicht in $\beta\left(Z_{n}\right)$ enthalten ist; von diesen Zahlen $p$ wählen wir - nur um etwas Bestimmtes festzusetzen - immer die kleinste $k(96)$ und definieren, da $Z_{n^{\prime}}$ nach 108 aus $Z_{n}$ und $n^{\prime}$ zusammengesetzt ist, eine Abbildung $\gamma$ von $Z_{n^{\prime}}$ dadurch, daß für alle in $Z_{n}$ enthaltenen Zahlen $m$ das Bild $\gamma(m)=\beta(m)$, und außerdem $\gamma\left(n^{\prime}\right)=\alpha_{n^{\prime}}(k)$ sein soll; diese, offenbar ähnliche, Abbildung $\gamma$ von $Z_{n^{\prime}}$ in $\Sigma$ sehen wir nun als ein Bild $\theta(\beta)$ der Abbildung $\beta$ an, und hierdurch ist eine Abbildung $\theta$ des Systems $\Omega$ in sich selbst vollständig definiert. Nachdem die in 126 genannten Dinge $\Omega$ und $\theta$ bestimmt sind, wählen wir endlich für das mit $\omega$ bezeichnete Element von $\Omega$ die gegebene Abbildung $\alpha_{1}$; hierdurch ist nach 126 eine Abbildung $\psi$ der Zahlenreihe $N$ in $\Omega$ bestimmt, welche, wenn wir das zugehörige Bild einer beliebigen Zahl $n$ nicht mit $\psi(n)$, sondern mit $\psi_{n}$ bezeichnen, den Bedingungen

$$
\begin{aligned}
\text { II. } & \psi_{1}=\alpha_{1}, \\
\text { III. } & \psi_{n^{\prime}}=\theta\left(\psi_{n}\right)
\end{aligned}
$$


genügt. Durch vollständige Induktion (80) ergibt sich zunächst, daß $\psi_{n}$ eine ähnliche Abbildung von $Z_{n}$ in $\Sigma$ ist; denn

o. dies ist zufolge II wahr für $n=1$, und

б. wenn diese Behauptung für eine Zahl $n$ zutrifft, so folgt aus III und aus der Art des oben beschriebenen Überganges $\theta$ von $\beta$ zu $\gamma$, daß die Behauptung auch für die folgende Zahl $n^{\prime}$ gilt, w. z. b. w. Hierauf beweisen wir ebenfalls durch vollständige Induktion (80), daß, wenn $m$ irgendeine Zahl ist, die oben angekündigte Eigenschaft

$$
\psi_{n}(m)=\psi_{m}(m)
$$

wirklich allen Zahlen $n$ zukommt, welche $\geqq m$ sind, also nach 93 , 74 der Kette $m_{0}$ angehören; in der Tat,

o. dies leuchtet unmittelbar ein für $n=m$, und

б. wenn diese Eigenschaft einer Zahl $n$ zukommt, so folgt wieder aus III und der Beschaffenheit von $\theta$, daß sie auch der Zahl $n^{\prime}$ zukommt, w. z. b. w. Nachdem auch diese besondere Eigenschaft unserer neuen Reihe von Abbildungen $\psi_{n}$ festgestellt ist, können wir unseren Satz leicht beweisen. Wir definieren eine Abbildung $\chi$ der Zahlenreihe $N$, indem wir jeder Zahl $n$ das Bild $\chi(n)=\psi_{n}(n)$ entsprechen lassen; offenbar sind (nach 21) alle Abbildungen $\psi_{n}$ in dieser einen Abbildung $\chi$ enthalten. Da $\psi_{n}$ eine Abbildung von $Z_{n}$ in $\Sigma$ war, so folgt zunächst, daß die Zahlenreihe $N$ durch $\chi$ ebenfalls in $\Sigma$ abgebildet wird, also $\chi(N) 3 \Sigma$ ist. Sind ferner $m, n$ verschiedene Zahlen, so darf man der Symmetrie wegen nach 90 annehmen, es sei $m<n$; dann ist nach dem Obigen $\chi(m)=\psi_{m}(m)=\psi_{n}(m)$ und $\chi(n)=\psi_{n}(n)$; da aber $\psi_{n}$ eine ähnliche Abbildung von $Z_{n}$ in $\Sigma$ war, und $m, n$ verschiedene Elemente von $Z_{n}$ sind, so ist $\psi_{n}(m)$ verschieden von $\psi_{n}(n)$, also auch $\chi(m)$ verschieden von $\chi(n)$, d. h. $\chi$ ist eine ähnliche Abbildung von $N$. Da ferner $N$ ein unendliches System ist (71), so gilt nach 67 dasselbe von dem ihm ähnlichen System $\chi(N)$ und nach 68 , weil $\chi(N)$ Teil von $\Sigma$ ist, auch von $\Sigma$, w. z.b.w.

160. Satz. Ein System $\Sigma$ ist endlich oder unendlich, je nachdem es ein ihm ähnliches System $Z_{n}$ gibt oder nicht gibt.

Bewe is. Wenn $\Sigma$ endlich ist, so gibt es nach 159 Systeme $Z_{n}$, welche nicht ähnlich abbildbar in $\Sigma$ sind; da nach 102 das System $Z_{1}$ 
aus der einzigen Zahl 1 besteht und folglich in jedem System ähnlich abbildbar ist, so muß die kleinste Zahl $k(96)$, der ein in $\Sigma$ nicht ähnlich abbildbares System $Z_{k}$ entspricht, verschieden von 1, also (nach 78) $=n^{\prime}$ sein, und da $n<n^{\prime}$ ist (91), so gibt es eine ähnliche Abbildung $\psi$ von $Z_{n}$ in $\Sigma$; wäre nun $\psi\left(Z_{n}\right)$ nur ein echter Teil von $\Sigma$, gäbe es also ein Element $\alpha$ in $\Sigma$, welches nicht in $\psi\left(Z_{n}\right)$ enthalten ist, so könnte man, da $Z_{n^{\prime}}=\mathfrak{M}\left(Z_{n}, n^{\prime}\right)$ ist (108), diese Abbildung $\psi$ zu einer ähnlichen Abbildung $\psi$ von $Z_{n^{\prime}}$ in $\Sigma$ erweitern, indem man $\psi\left(n^{\prime}\right)=\alpha$ setzte, während doch nach unserer Annahme $Z_{n^{\prime}}$ nicht ähnlich abbildbar in $\Sigma$ ist. Mithin ist $\psi\left(Z_{n}\right)=\Sigma$, d. h. $Z_{n}$ und $\Sigma$ sind ähnliche Systeme. Umgekehrt, wenn ein System $\Sigma$ einem System $Z_{n}$ ähnlich ist, so ist $\Sigma$ nach 119, 67 endlich, w. z. b.w.

161. Erklärung. Ist $\Sigma$ ein endliches System, so gibt es nach 160 eine, und nach 120,33 auch nur eine einzige Zahl $n$, welcher ein dem Systeme $\Sigma$ ähnliches System $Z_{n}$ entspricht; diese Zahl $n$ heißt die Anzahl der in $\Sigma$ enthaltenen Elemente (oder auch der Grad des Systems $\Sigma$ ), und man sagt, $\Sigma$ bestehe aus oder sei ein System von $n$ Elementen, oder die Zahl $n$ gebe an, wie viele Elemente in $\Sigma$ enthalten sind*). Wenn die Zahlen benutzt werden, um diese bestimmte Eigenschaft endlicher Systeme genau auszudrücken so heißen sie Kardinalzahlen. Sobald eine bestimmte ähnliche Abbildung $\psi$ des Systems $Z_{n}$ gewählt ist, vermöge welcher $\psi\left(Z_{n}\right)=\Sigma$ wird, so entspricht jeder in $Z_{n}$ enthaltenen Zahl $m$ (d.h. jeder Zahl $m$, welche $\leqq n$ ist) ein bestimmtes Element $\psi(m)$ des Systems $\Sigma$, und rückwärts entspricht nach 26 jedem Elemente von $\Sigma$ durch die umgekehrte Abbildung $\bar{\psi}$ eine bestimmte Zahl $m$ in $Z_{n}$. Sehr oft bezeichnet man alle Elemente von $\Sigma$ mit einem einzigen Buchstaben, z. B. $\alpha$, dem man die unterscheidenden Zahlen $m$ als Zeiger anhängt, so daß $\psi(m)$ mit $\alpha_{m}$ bezeichnet wird. Man sagt auch, diese Elemente seien gezählt und durch $\psi$ in bestimmter Weise geordnet, und nennt $\alpha_{m}$ das $m$-te Element von $\Sigma$; ist $m<n$, so heißt $\alpha_{m^{\prime}}$ das auf $\alpha_{m}$ folgende Element, und $\alpha_{n}$ heißt das letzte Element. Bei diesem Zählen der Elemente treten daher die Zahlen $m$ wieder als Ordinalzahlen auf (73).

*) Der Deutlichkeit und Einfachheit wegen beschränken wir im folgenden den Begriff der Anzahl durchaus auf endliche Systeme; wenn wir daher von einer Anzahl gewisser Dinge sprechen, so soll damit immer schon ausgedrückt sein, da das System, dessen Elemente diese Dinge sind, ein endliches ist. 
162. Satz. Alle einem endlichen Systeme ähnlichen Systeme besitzen dieselbe Anzahl von Elementen.

Der Beweis folgt unmittelbar aus 33, 161.

163. Satz. Die Anzahl der in $Z_{n}$ enthaltenen, d. h. derjenigen Zahlen, welche $\leqq n$ sind, ist $n$.

Beweis. Denn nach 32 ist $Z_{n}$ sich selbst ähnlich.

164. Satz. Besteht ein System aus einem einzigen Element, so ist die Anzahl seiner Elemente $=1$, und umgekehrt.

Der Beweis folgt unmittelbar aus 2, 26, 32, 102, 161.

165. Satz. Ist $T$ echter Teil eines endlichen Systems $\Sigma$, so ist die Anzahl der Elemente von $T$ kleiner als diejenige der Elemente von $\Sigma$.

Beweis. Nach 68 ist $T$ ein endliches System, also ähnlich einem System $Z_{m}$, wo $m$ die Anzahl der Elemente von $T$ bedeutet; ist ferner $n$ die Anzahl der Elemente von $\Sigma$, also $\Sigma$ ähnlich $Z_{n}$, so ist $T$ nach 35 einem echten Teile $E$ von $Z_{n}$ ähnlich, und nach 33 sind auch $Z_{m}$ und $E$ einander ähnlich; wäre nun $n \leqq m$, also $Z_{n} 3 Z_{m}$, so wäre $E$ nach 7 auch echter Teil von $Z_{m}$, und folglich $Z_{m}$ ein unendliches System, was dem Satze 119 widerspricht; mithin ist (nach 90) $m<n$, w. z. b. w.

166. Satz. Ist $\Gamma=\mathfrak{M}(B, \gamma)$, wo $B$ ein System von $n$ Elementen und $\gamma$ ein nicht in $B$ enthaltenes Element von $\Gamma$ bedeutet, so besteht $\Gamma$ aus $n^{\prime}$ Elementen.

Beweis. Denn wenn $B=\psi\left(Z_{n}\right)$ ist, wo $\psi$ eine ähnliche $\mathrm{Ab}$ bildung von $Z_{n}$ bedeutet, so läßt sich dieselbe nach $105,108 \mathrm{zu}$ einer ähnlichen Abbildung $\psi$ von $Z_{n^{\prime}}$ erweitern, indem man $\psi\left(n^{\prime}\right)=\gamma$ setzt, und zwar wird $\psi\left(Z_{n^{\prime}}\right)=\Gamma$, w. z. b. w.

167. Satz. Ist $\gamma$ ein Element eines aus $n^{\prime}$ Elementen bestehenden Systems $\boldsymbol{\Gamma}$, so ist $n$ die Anzahl aller anderen Elemente von $\boldsymbol{\Gamma}$.

Beweis. Denn wenn $B$ den Inbegriff aller von $\gamma$ verschiedenen Elemente in $\Gamma$ bedeutet, so ist $\Gamma=\mathfrak{M}(B, \gamma)$; ist nun $b$ die Anzahl der Elemente des endlichen Systems $B$, so ist nach dem vorhergehenden Satze $b^{\prime}$ die Anzahl der Elemente von $\Gamma$, also $=n^{\prime}$, woraus nach 26 auch $b=n$ folgt, w. z. b. w.

168. Satz. Besteht $A$ aus $m$, und $B$ aus $n$ Elementen, und haben $A$ und $B$ kein gemeinsames Element, so besteht $\mathfrak{M}(A, B)$ aus $m+n$ Elementen. 
Beweis durch vollständige Induktion (80). Denn

@. der Satz ist wahr für $n=1$ zufolge $166,164,135$. II.

б. Gilt der Satz für eine Zahl $n$, so gilt er auch für die folgende Zahl $n^{\prime}$. In der Tat, wenn $\Gamma$ ein System von $n^{\prime}$ Elementen ist, so kann man (nach 167) $\Gamma=\mathfrak{M}(B, \gamma)$ setzen, wo $\gamma$ ein Element und $B$ das System der $n$ anderen Elemente von $\Gamma$ bedeutet. Ist nun $A$ ein System von $m$ Elementen, deren jedes nicht in $\Gamma$, also auch nicht in $B$ enthalten ist, und setzt man $\mathfrak{M}(A, B)=\Sigma$, so ist nach unserer Annahme $m+n$ die Anzahl der Elemente von $\Sigma$, und da $\gamma$ nicht in $\Sigma$ enthalten ist, so ist nach 166 die Anzahl der in $\mathfrak{M}(\Sigma, \gamma)$ enthaltenen Elemente $=(m+n)^{\prime}$, also (nach 135. III) $=m+n^{\prime}$; da aber nach 15 offenbar $\mathfrak{M}(\Sigma, \gamma)=\mathfrak{M}(A, B, \gamma)=\mathfrak{M}(A, \Gamma)$ ist, so ist $m+n^{\prime}$ die Anzahl der Elemente von $\mathfrak{M}(A, \Gamma)$, w. z. b. w.

169. Satz. Sind $A, B$ endliche Systeme von beziehungsweise $m, n$ Elementen, so ist $\mathfrak{M}(A, B)$ ein endliches System, und die Anzahl seiner Elemente ist $\leqq m+n$.

Beweis. Ist $B 3 A$, so ist $\mathfrak{M}(A, B)=A$, und die Anzahl $m$ der Elemente dieses Systems ist (nach 142) $<m+n$, wie behauptet war. Ist aber $B$ kein Teil von $A$, und $T$ das System aller derjenigen Elemente von $B$, welche nicht in $A$ enthalten sind, so ist nach 165 deren Anzahl $p \leqq n$, und da offenbar

$$
\mathfrak{M}(A, B)=\mathfrak{M}(A, T)
$$

ist, so ist nach 143 die Anzahl $m+p$ der Elemente dieses Systems $\leqq m+n$, w. z. b. w.

170. Satz. Jedes aus einer Anzahl $n$ von endlichen Systemen zusammengesetzte System ist endlich.

Beweis durch vollständige Induktion (80). Denn

@. der Satz ist nach 8 selbstrerständlich für $n=1$.

б. Gilt der Satz für eine Zahl $n$, und ist $\Sigma$ zusammengesetzt aus $n^{\prime}$ endlichen Systemen, so sei $A$ eines dieser Systeme und $B$ das aus allen übrigen zusammengesetzte System; da deren Anzahl (nach 167) $=n$ ist, so ist nach unserer Annahme $B$ ein endliches System. Da nun offenbar $\Sigma=\mathfrak{M}(A, B)$ ist, so folgt hieraus und aus 169 , daß auch $\Sigma$ ein endliches System ist, w. z. b. w.

171. Satz. Ist $\psi$ eine unähnliche Abbildung eines endlichen Systems $\Sigma$ von $n$ Elementen, so ist die Anzahl der Elemente des Bildes $\psi(\Sigma)$ kleiner als $n$. 
Beweis. Wählt man von allen denjenigen Elementen von $\Sigma$, welche ein und dasselbe Bild besitzen, immer nur ein einziges nach Belieben aus, so ist das System $T$ aller dieser ausgewählten Elemente offenbar ein echter Teil von $\Sigma$, weil $\psi$ eine unähnliche Abbildung von $\Sigma$ ist (26). Zugleich leuchtet aber ein, daß die (nach 21) in $\psi$ enthaltene Abbildung dieses Teils $T$ eine ähnliche, und daß $\psi(T)=\psi(\Sigma)$ ist; mithin ist das System $\psi(\Sigma)$ ähnlich dem echten Teil $T$ von $\Sigma$, und hieraus folgt unser Satz nach 162, 165.

172. Schlußbemerkung. Obgleich soeben bewiesen ist, daß die Anzahl $m$ der Elemente von $\psi(\Sigma)$ kleiner als die Anzahl $n$ der Elemente von $\Sigma$ ist, so sagt man in manchen Fällen doch gern, die Anzahl der Elemente von $\psi(\Sigma)$ sei $=n$. Natürlich wird dann das Wort Anzahl in einem anderen als dem bisherigen Sinne (161) gebraucht; ist nämlich $\alpha$ ein Element von $\Sigma$, und $a$ die Anzahl aller derjenigen Elemente von $\Sigma$, welche ein und dasselbe Bild $\psi(\alpha)$ besitzen, so wird letzteres als Element von $\psi(\Sigma)$ häufig doch noch als Vertreter von $a$ Elementen aufgefaßt, die wenigstens ihrer Abstammung nach als verschieden voneinander angesehen werden können, und wird demgemäß als $a$-faches Element von $\psi(\Sigma)$ gezählt. Man kommt auf diese Weise zu dem in vielen Fällen sehr nützlichen Begriffe von Systemen, in denen jedes Element mit einer gewissen Häufigkeitszahl ausgestattet ist, welche angibt, wie oft dasselbe als Element des Systems gerechnet werden soll. Im obigen Falle würde man z. B. sagen, daß $n$ die Anzahl der in diesem Sinne gezählten Elemente von $\psi(\Sigma)$ ist, während die Anzahl $m$ der wirklich verschiedenen Elemente dieses Systems mit der Anzahl der Elemente von $T$ übereinstimmt. Ähnliche Abweichungen von der ursprünglichen Bedeutung eines Kunstausdrucks, die nichts anderes sind als Erweiterungen der ursprünglichen Begriffe, treten sehr häufig in der Mathematik auf; doch liegt es nicht im Zweck dieser Schrift, näher hierauf einzugehen.

\section{Erläuterungen zur vorstehenden Abhandlung*).}

"Was sind und was sollen die Zahlen?" ist in zwei Richtungen bahnbrechend geworden, für die Grundlagenforschung und für die axiomatische Mengenlehre. Auf die Bedeutung für die Grundlagenforschung hat erst neuerdings Hilbert wieder hingewiesen (Math. Ann. 104); eine eingehende von E. Zermelo stammende Analyse der Schrift findet sich in dem Nachruf von Landau (Gött. Nachr. 1917). Wie stark die axiomatische Mengenlehre durch Dedekind beeinflußt ist, zeigt ein

*) Vgl. auch die Erläuterungen zu LX und LXII. 
Vergleich mit den Zermeloschen Axiomen (Math. Ann. 65), die teilweise direkt aus den „Erklärungen" Dedekinds ( 1 der Schrift) übernommen sind. Daß dabei das "Axiom des Unendlichen" postuliert werden mußte, da der Beweisversuch Dedekinds (66) auf dem widerspruchsvollen Begriff der "Menge alles Denkbaren" beruht, ist bekannt; ebenso, daß in die Dedekindschen Überlegungen das Auswahlpostulat hineinspielt (159). Auch der zweite Zermelosche Beweis des Wohlordnungssatzes kann als eine Übertragung des hier gegebenen Beweises für die Möglichkeit der vollständigen Induktion auf die transfinite Induktion angesehen werden; dabei mußte allerdings im Transfiniten schon hier das Auswahlaxiom den übrigen, von Dedekind implizit benutzten Axiomen zugefügt werden. Dedekind konnte es für die gewöhnliche vollständige Induktion umgehen, dadurch, daß er die in die Definition des Unendlichen eingehende Abbildung zur Verfügung hatte. Der über den "Beweis“ durch vollständige Induktion hinausgehende Satz von der „Definition“ durch vollständige Induktion (126) ist für das Transfinite scharf herausgearbeitet bei J. v. Neumann (Math. Ann. 99). Der Satz findet insbesondere Verwendung in der Algebra unendlicher Bereiche, entsprechend wie Dedekind die Rechnungsregeln der ganzen Zahlen vermöge Definition durch vollständige Induktion erhält.

Noether. 